\title{
Brain-Computer Interfaces for Assessment and Communication in Disorders of Consciousness
}

Citation for published version (APA):

Guger, C., Sorger, B., Noirhomme, Q., Naci, L., Monti, M. M., Real, R., Pokorny, C., Veser, S., Lugo, Z., Quitadamo, L., Lesenfants, D., Risetti, M., Formisano, R., Toppi, J., Astolfi, L., Emmerling, T., Heine, L., Erlbeck, H., Horki, P., ... Cincotti, F. (2014). Brain-Computer Interfaces for Assessment and

Communication in Disorders of Consciousness. In G. R. Naik, \& Y. Guo (Eds.), Emerging Theory and Practice in Neuroprosthetics (pp. 181-214). IGI Global Publishing. https://doi.org/10.4018/978-1-46666094-6.ch010

Document status and date:

Published: 01/01/2014

DOI:

10.4018/978-1-4666-6094-6.ch010

Document Version:

Publisher's PDF, also known as Version of record

Document license:

Taverne

Please check the document version of this publication:

- A submitted manuscript is the version of the article upon submission and before peer-review. There can be important differences between the submitted version and the official published version of record.

People interested in the research are advised to contact the author for the final version of the publication, or visit the DOI to the publisher's website.

- The final author version and the galley proof are versions of the publication after peer review.

- The final published version features the final layout of the paper including the volume, issue and page numbers.

Link to publication

\footnotetext{
General rights Owners
rights.

- You may freely distribute the URL identifying the publication in the public portal. please follow below link for the End User Agreement:

www.umlib.nl/taverne-license

Take down policy

If you believe that this document breaches copyright please contact us at:

repository@maastrichtuniversity.nl

providing details and we will investigate your claim.
}

Copyright and moral rights for the publications made accessible in the public portal are retained by the authors and/or other copyright owners and it is a condition of accessing publications that users recognise and abide by the legal requirements associated with these

- Users may download and print one copy of any publication from the public portal for the purpose of private study or research.

- You may not further distribute the material or use it for any profit-making activity or commercial gain

If the publication is distributed under the terms of Article $25 \mathrm{fa}$ of the Dutch Copyright Act, indicated by the "Taverne" license above, 
See discussions, stats, and author profiles for this publication at: https://www.researchgate.net/publication/259013574

\section{Brain-Computer Interfaces for Assessment and Communication in Disorders of Consciousness}

Chapter · January 2013

DOI: 10.4018/978-1-4666-6094-6.ch010

CITATIONS

12

28 authors, including:

Christoph Guger

g.tec medical engineering $\mathrm{GmbH}$

369 PUBLICATIONS 8,850 CITATIONS

SEE PROFILE

Quentin Noirhomme

Brain Innovation, Maastricht, Netherlands

132 PUBLICATIONS 6,239 CITATIONS

SEE PROFILE

Some of the authors of this publication are also working on these related projects:

Project Brain connectivity analysis View project

Project FeelYourReach View project
READS

1,499

Bettina Sorger

Maastricht University

100 PUBLICATIONS 3,185 CITATIONS

SEE PROFILE

Lorina $\mathrm{Naci}$

Trinity College Dublin

55 PUBLICATIONS 1,334 CITATIONS

SEE PROFILE 


\section{Emerging Theory and Practice in Neuroprosthetics}

Ganesh R. Naik

University of Technology Sydney (UTS), Australia

Yina Guo

Taiyuan University of Science and Technology, China

A volume in the Advances in Bioinformatics and

Biomedical Engineering (ABBE) Book Series 


\section{Detailed Table of Contents}

Preface xvi

\section{Section 1 \\ Neuroprosthetics Theory and Model}

\section{Chapter 1}

Neuroprosthetics: Introduction 1

Ganesh R. Naik, University of Technology Sydney (UTS), Australia

In this chapter, Ganesh Naik explains the recent advances and applications of neuroprosthetics. Neuroprostheses use electric stimuli to stimulate neural structures, muscles, or receptors in order to support, augment, or partly restore the respective disordered or lost function. The objective is to help the patient to participate in everyday life. The use of a neural prosthesis can improve the quality of life of the person concerned. The future of neuroprosthetics is challenging as well as interesting as it deals with several latest technological advancements that connect both biology and technology together.

\section{Chapter 2}

Retinal Prosthetics. .8

Milan Djilas, INSERM/CNRS/UMPC, France

Serge Picaud, INSERM/CNRS/UMPC, France

In this chapter, Djilas and Picaud discuss neuroanatomical and current open issues of the retinal prosthetics, such as implant placement, biocompatibility, electrode design, and safety. They first briefly introduce the neuroanatomical basis for vision and explain how the retina processes visual information. Pathology of the retina and the conditions that cause photoreceptors degeneration and lead to blindness are then given, followed by the main part of the chapter in which authors present an overview of the concept of restoring vision with visual prosthetics. The focus is specifically on retinal prostheses and electrical stimulation parameters used with these devices. Both in vitro and in vivo animal studies from the last decade are surveyed, together with the latest results from human trials conducted in multiple research centres worldwide. In the final section, the authors give their opinion on the future development and perspectives of the retinal prosthetics research. 


\section{Chapter 3}

Sensors for Motor Neuroprosthetics: Current Applications and Future Directions

Emilia Ambrosini, Politecnico di Milano, Italy

Noelia Chia Bejarano, Politecnico di Milano, Italy

Alessandra Pedrocchi, Politecnico di Milano, Italy

In this chapter, Ambrosini, Bejarano, and Pedrocchi summarize the state of the art of sensors used in Functional Electrical Stimulation (FES) and motor neuroprostheses. Clinical applications of FES provide both functional and therapeutic benefits. To enhance the functionality of FES systems and to improve the control of the activated muscles through open-loop or feedback controllers, solutions to gather information about the status of the system in real time and to easily detect the intention of the subject have to be optimized. These sensors can be classified in two categories: sensors of biological signals and sensors of non-biological signals. Here, the authors report definitions, advantages, and disadvantages for each sensor and also explain guidelines to compare sensors for the design of motor neuroprostheses.

\section{Chapter 4}

Models of Cooperation between Medical Specialists and Biomedical Engineers in

Neuroprosthetics

Emilia Mikołajewska, Military Clinical Hospital No. 10 and Polyclinic, Poland

Dariusz Mikołajewski, Kazimierz Wielki University, Poland \& Nicolaus Copernicus

University, Poland

In this chapter, Mikołajewska and Mikołajewski answer the question, how can biomedical engineers be incorporated into research and clinical practice in neuroprosthetics considering the various factors, necessary changes in educational processes, ethical issues, and associated organizational problems? Current models of education and cooperation within interdisciplinary therapeutic teams only concern medical specialists. The development of novel technologies associated with neuroprosthetics and their clinical applications needs and interdisciplinary knowledge, including not only medical sciences, but IT, biomedical engineering, biocybernetics, and robotics. The variability of possible neurological deficits, interventions, and even scales-from nanotechnology up to rehabilitation robots and brain-computerinterface controlled exoskeletons as whole-body neuroprostheses-make this task very difficult. 


\section{Chapter 5}

Are We the Robots? Man-Machine Integration

Iolanda Pisotta, Neurological and Spinal Cord Injury Rehabilitation Department A and

CaRMA Lab, IRCCS Fondazione S. Lucia, Italy \& University of Rome "La Sapienza”, Italy

Silvio Ionta, Swiss Federal Institute of Technology Zurich (ETHZ), Switzerland

In this chapter, Pisotta and Ionta review the hurdles involved in making the Brain-Machine Interfaces (BMIs) a reality. Humans experience and interact with the world through their body. One of the most important features of the human is the interaction between mind and body. Since the original demonstration that electrical activity of the cortical neurons can be employed to directly control a robotic device, the research on the so-called BMIs has impressively grown. For example, current BMIs dedicated to both experimental and clinical studies can translate raw neuronal signals into computational commands to reproduce reaching or grasping in artificial actuators. These developments hold promise for the restoration of limb mobility in paralyzed individuals. Before this goal can be achieved, several hurdles have to be overcome, including developments in real-time computational algorithms and in designing fully implantable and biocompatible devices. Future investigations will have to address the best solutions

for restoring sensation to the prosthetic limb, which still remains a major challenge to full integration of the limb into the user's self-image.

\section{Chapter 6}

Neuroprostheses as an Element of an Eclectic Approach to Intervention in Neurorehabilitation......101 Emilia Mikołajewska, Military Clinical Hospital No. 10 and Polyclinic, Poland

In this chapter, Mikołajewska investigates the extent to which the available opportunities in BrainComputer Interfaces (BCI) and Neuroprostheses (NP) are being exploited, including current and potential future applications of NP within an eclectic approach to intervention in neurorehabilitation. Improvements in the effectiveness of contemporary neurorehabilitation emphasize the need for a shift from a specific approach to intervention to an eclectic approach to intervention. The novel strategies of BCI and NP application in an eclectic approach to intervention may be regarded as leading the way in clinical practice development. There is a limited amount of evidence both in the areas of theoretical principles and clinical applications, but it seems the application of various rehabilitation methods and techniques may effectively support the outcomes of the BCI's and NP's use. 


\section{Chapter 7}

Chances for and Limitations of Brain-Computer Interface use in Elderly People. 116

Emilia Mikołajewska, Military Clinical Hospital No. 10 and Polyclinic, Poland

Dariusz Mikołajewski, Kazimierz Wielki University, Poland \& Nicolaus Copernicus

University, Poland

Tomasz Komendziński, Nicolaus Copernicus University, Poland

Joanna Dreszer-Drogorób, Nicolaus Copernicus University, Poland

Monika Lewandowska, Institute of Physiology and Pathology of Hearing, Poland

Tomasz Wolak, Institute of Physiology and Pathology of Hearing, Poland

In this chapter, Mikołajewska et al. investigate the available opportunities and limitations in usage of Assistive Technology (AT) devices for elderly people, including medical, technical, psychological, societal, ethical, and legal issues. Recent demographic prognoses show tendencies toward significant increase in number of elderly people, especially in developed countries. This makes geriatric therapy, rehabilitation, and care difficult, especially with maintaining the highest quality of life and independence in activities of daily living as long as possible. Lack of specialized personnel and financial shortages may cause increased application of Assistive Technology (AT) and associated control devices. The most advanced current devices for diagnosis, communication, and control purposes are perceived Brain-Computer Interfaces (BCIs). BCIs are regarded as novel solutions offering another breakthrough in everyday life, care, therapy, and rehabilitation in patients with severe sensory and neuropsychological deficits. However, particular issues in the area of BCIs use in elderly people should be emphasized, including influence of neurodegenerative disorders accompanied with secondary changes resulting from other medical problems (e.g. heart diseases, hypertension, diabetes mellitus, and osteoporosis), co-occurence of various drug therapies, etc.

\section{Section 2 Neuroprosthetics for BCI and Other Applications}

\section{Chapter 8}

Review of Applications for Wireless Brain-Computer Interface Systems.... 128

Soogil Woo, Gwangju Institute of Science and Technology, South Korea

Younghak Shin, Gwangju Institute of Science and Technology, South Korea

Seungchan Lee, Gwangju Institute of Science and Technology, South Korea

Heung-No Lee, Gwangju Institute of Science and Technology, South Korea

In this chapter, Soogil Woo et al. review the research trends for wireless Brain-Computer Interface (BCI) systems, as well as their current and anticipated applications. Wireless BCI systems have clear advantages, when compared to wired BCI systems, in that they have simpler shapes and can be convenient and portable devices. Recent wireless BCI applications attempt to help people live more conveniently in many areas of life: medical engineering, rehabilitation, and everyday life. 


\section{Chapter 9}

Lower-Limb Neuroprostheses: Restoring Walking after Spinal Cord Injury 153

Monzurul Alam, The Hong Kong Polytechnic University, Hong Kong

Jufang He, The Hong Kong Polytechnic University, Hong Kong \& City University of Hong Kong, Hong Kong

In this chapter, Alam and He discuss available therapies for the rehabilitation of Spinal Cord Injury (SCI) paraplegics and some new potential interventions that still require clinical tests. They also propose brain-machine-spinal cord interface as a future neuroprosthesis following motor complete SCI. Regaining lower-limb functionality such as walking is one of the highest priorities among all the disabilities of paraplegics following SCI. Though the ultimate recovery would be repairing or regenerating new axons across the spinal lesion (potentially by stem cells or other transplants and neurotropic factors), challenges to achieve this as well as recent technological advancements demand the development of new neuroprosthetic devices to restore such motor functions following the injuries.

\section{Chapter 10}

Brain-Computer Interfaces for Assessment and Communication in Disorders of Consciousness ......181

Christoph Guger, Guger Technologies, Austria

Bettina Sorger, Maastricht University, The Netherlands

Quentin Noirhomme, University of Liege, Belgium

Lorina Naci, University of Western Ontario, Canada

Martin M. Monti, University of California, Los Angeles, USA

Ruben Real, Universität Würzburg, Germany

Christoph Pokorny, Graz University of Technology, Austria

Sandra Veser, University of Tübingen, Germany

Zulay Lugo, University of Liège, Belgium

Lucia Quitadamo, University of Tor Vergata, Italy

Damien Lesenfants, Université de Liège, Belgium

Monica Risetti, Fondazione Santa Lucia, Italy

Rita Formisano, Ospedale di Riabilitazione Fondazione Santa Lucia, Italy

Jlenia Toppi, University of Rome "La Sapienza," Italy

Laura Astolfi, Università di Roma "La Sapienza," Italy

Thomas Emmerling, Maastricht University, The Netherlands

Lizette Heine, Maastricht University, The Netherlands

Helena Erlbeck, Universität Würzburg, Germany

Petar Horki, Graz University of Technology, Austria

Boris Kotchoubey, University of Tübingen, Germany

Luigi Bianchi, "Tor Vergata” University of Rome, Italy

Donatella Mattia, Fondazione Santa Lucia, Italy

Rainer Goebel, Maastricht University, The Netherlands

Adrian M. Owen, Western University, Canada

Frederic Pellas, Association Locked-In Syndrome, France

Gernot Müller-Putz, Graz University of Technology, Austria

Steven Laureys, University of Liege, Belgium

Andrea Kübler, Universität Würzburg, Germany 
In this chapter, Guger et al. discuss current methods and problems associated with Disorders of Consciousness (DOC) and highlight the possible solutions to the same. Many patients with DOC are misdiagnosed for a variety of reasons. These patients typically cannot communicate. Because such patients are not provided with the needed tools, one of their basic human needs remains unsatisfied, leaving them truly locked in their bodies. This chapter first reviews current methods and problems of diagnoses and assistive technology for communication, supporting the view that advances in both respects are needed for patients with DOC. The authors also discuss possible solutions to these problems and introduce emerging developments based on EEG (Electroencephalography), fMRI (Functional Magnetic Resonance Imaging), and fNIRS (Functional Near-Infrared Spectroscopy) that have been validated with patients and healthy volunteers.

\section{Chapter 11}

Assistive Technology for Cognition: An Updated Review

Catherine Best, University of Stirling, UK

Brian O'Neill, Graham Anderson House, UK

Alex Gillespie, London School of Economics, UK

In this chapter, Best, O'Neill, and Gillespie review the use of assistive technology in health and social care for people with cognitive impairment. This review updates their previous reviews on this topic and reflects on how their conceptualization of Assistive Technology for Cognition (ATC) in terms of function (reminding, alerting, micro prompting, distracting, storing, displaying, navigating, and biofeedback), as opposed to the type of technology (mobile phone, desktop computer, etc.), and fits with recent developments in this field. The authors highlight the growing number of context-aware prompting devices and the move to train people with cognitive impairment to use everyday technology such as mobile phones. They also make a distinction between ATC, which augments or supplants cognitive functions, and outline avenues for future research.

\section{Chapter 12}

Brain-Computer Interfaces for Control of Upper Extremity Neuroprostheses in Individuals with High Spinal Cord Injury

Rüdiger Rupp, Heidelberg University Hospital, Germany

Martin Rohm, Heidelberg University Hospital, Germany

Matthias Schneiders, Heidelberg University Hospital, Germany

In this chapter, Rupp et al. provide an overview of the current state of the art of Brain-Computer Interface (BCI) controlled upper-extremity neuroprostheses and describe the challenges and promises for the future. For individuals with tetraplegia, restoring limited or missing grasping function is the highest priority. In patients with high Spinal Cord Injury (SCI) and a lack of surgical options, restricted upper extremity function can be improved with the use of neuroprostheses based on Functional Electrical Stimulation (FES). Grasp neuroprostheses with different degrees of complexity and invasiveness exist, although few models are available for routine clinical application. Hybrid systems combining FES with orthoses hold promise for restoring completely lost upper extremity function. Novel user interfaces integrating biosignals from several sources are needed to make full use of the many degrees of freedom of hybrid neuroprostheses. Motor Imagery (MI)-based BCIs are an emerging technology that may serve as a valuable adjunct to traditional control interfaces. 


\section{Chapter 13}

Practical Artifact Removal Brain-Computer Interface System: Application to Neuroprosthetics......265

Wei-Yen Hsu, National Chung Cheng University, Taiwan

In this chapter, Wei-Yen Hsu presents a practical artifact removal Brain-Computer Interface (BCI) system for single-trial Electroencephalogram (EEG) data. Initially, Independent Component Analysis (ICA) combined with the use of a correlation coefficient is used to remove the EOG artifacts automatically, which can further improve classification accuracy. The features are then extracted from wavelet transform data by means of the proposed modified fractal dimension. Finally, Support Vector Machine (SVM) is used for the classification. When compared with the results obtained without using the EOG signal elimination, the proposed BCI system achieves promising results that will be effectively applied in neuroprosthetics applications.

Related References 278

Compilation of References 312

About the Contributors 390

Index 404 


\section{About the Contributors}

Ganesh R. Naik received B.E. degree in Electronics and Communication Engineering from the University of Mysore, India, in 1997, M.E. degree in Communication and Information Engineering from Griffith University, Brisbane, Australia, in 2002, and the PhD degree in the area of Electronics Engineering, specialised in Biomedical Engineering and Signal Processing from RMIT University, Melbourne, Australia, in 2009. He is currently Chancellor's Post Doctoral Research Fellow at Faculty of Engineering and Information Technology (FEIT), UTS. As an early career researcher, he has edited 9 books, authored more than 80 papers in peer-reviewed journals, conferences, and book chapters over the last 5 years. His research interests include EMG signal processing, Pattern Recognition, Blind Source Separation (BSS) techniques, Biomedical Signal Processing, Human-Computer Interface (HCI), and Audio Signal Processing. Currently, he serves as an associate editor for two Springer journals (Circuits, Systems, and Signal Processing and Australasian Physical \& Engineering Sciences in Medicine). He is also a reviewer and member of editorial board in several reputed journals. He is a recipient of the Baden-Württemberg Scholarship from the University of Berufsakademie, Stuttgart, Germany (2006-2007). In 2010, Dr. Naik is awarded with ISSI overseas fellowship from skilled Institute Victoria, Australia.

Yina Guo is an associate professor at the Department of Electronic and Communication, Taiyuan University of Science and Technology, Taiyuan, Shanxi, China. Her current research focuses on biosignal processing and machine learning. Yina Guo has taken charge of a General Program of National Natural Science Foundation of China, "Multiscale Analysis and Blind Source Separation of Single Channel Mixed Signals" (No.61301250) and several scientific projects of Shanxi province in last 5 years. She has obtained 3 patents in China: (1) "A Single Channel Blind Source Separation Method Based on sEMG in Human Body" (ZL201210114093.1), (2) "A sEMG Signal Collector in Human Body" (ZL201120232365.9), and (3) "A Seepage and Osmotic Pressure Monitoring Device" (ZL201010154776.0). In addition, she has published more than 30 refereed papers in last 5 years. She won the Merit Citation Class II and Merit Collective Citation Class I of Shanxi Province for Her Good Teaching Skill in 2010 and 2011, respectively, and Science and Technology Contribution Award of Shanxi Province in 2011. She is a member of the seventh congress in science and technology association of Taiyuan. 
Monzurul Alam received his $\mathrm{PhD}$ in Neuroprosthetics from The Hong Kong Polytechnic University and is currently working as a postdoctoral fellow at Dr. Edgerton's lab, University of California Los Angeles (UCLA). Both his PhD and postdoctoral research have been focused on Spinal Cord Injury (SCI) rehabilitation in rodent models. Electrophysiology, pharmacology, and electrical stimulation are the common techniques applied. Dr. Alam's major research interest is to understand the neural mechanisms underlying the recovery after injuries of the central and peripheral nervous system. He also applies modern engineering techniques to utilize this neural system for prosthetic applications.

Emilia Ambrosini graduated cum laude in Biomedical Engineering in 2007 and obtained a PhD Degree cum laude in Bioengineering in 2011 from Politecnico di Milano. In 2009, she was a visiting $\mathrm{PhD}$ student at the Control System Group of the Technische Universitat of Berlin. Since March 2011, she is a Research Fellow at Neuroengineering and medical robotics Laboratory (NearLab, www.biomed. polimi.it/nearlab), and she was involved in the European project MUNDUS (FP7 ICT- 2009.7.2). Her research interest is about the design and clinical translation of safe, simple, and immersive devices based on the integration of functional electrical stimulation and robotic systems for the rehabilitation and the daily life assistance of neurological patients. She is also interested on the assessment of neuroplasticity. She is co-author of 11 papers in peer-reviewed international journals.

Laura Astolfi received her MD in Electronic Engineering, summa cum laude, from University of Rome in 2003 and her $\mathrm{PhD}$ in Biomedical Engineering, with honors, from University of Bologna in 2007. She is currently an Assistant Professor at the Department of Computer, Control, and Management Engineering at Sapienza University of Rome and a Technical Manager at Fondazione Santa Lucia Hospital, Rome, Italy. She is author of 141 papers indexed in Scopus, of 3 books, and of more than 100 contributions to International Conferences and Schools. Her h-index is 18. She received several national and international awards for her scientific activity, among which the Best Under-40 Researcher Award at Sapienza University in 2010 and the Trainee Travel Award by the Human Brain Mapping Society in 2011. Her research includes functional connectivity, high resolution EEG source reconstruction, simultaneous recordings from multiple subjects (hyperscanning), consciousness, cognition, and social neuroscience.

Noelia Chia Bejarano received her Master of Science degree in Telecommunications Engineering in 2012 from the University of Seville, Spain. Prior to graduating, Noelia worked as a research assistant in the Electronic Engineering department at the University of Seville, and in 2009, she won a grant for a research internship at the Optics Institute "Daza de Valdés" of the Spanish National Research Council (CSIC), where she co-authored an article on Spectral Optical Coherence Tomography (sOCT). From 2011 to 2012, she was an exchange student at Politecnico di Milano, and a visiting student at the Neuroengineering and Medical Robotics Laboratory (NearLab). Since November 2012, Noelia is a PhD student in Bioengineering at Politecnico di Milano, where she is currently developing a rehabilitation system for neurological patients and older adults based on inertial sensors and functional electrical stimulation. 
Catherine Best has a BSc in Psychology and Neuroscience from the University of Manchester and a $\mathrm{PhD}$ from the University of Edinburgh, Division of Psychiatry. After graduating, she worked in services for people with long-term mental health conditions for 6 years. For the last 10 years, she has been involved in research in the field of health and social care. Her research interests are in the neuropsychology of neurodevelopmental and psychiatric disorders, interventions to promote independence well-being for people with long-term health problems, and assistive technology for cognition. Her methodological expertise and interests are in the conduct of systematic literature reviews and conducting research in vulnerable/difficult to recruit populations such as young people with developmental disorders and people with long-term mental health problems.

Luigi Bianchi is Assistant Professor at the "Dipartimento di Ingegneria Civile Ed Ingegneria Informatica" of the "Università di Roma Tor Vergata" Rome, Italy. He has a Ph.D. in Neurophysiology and is author of more than 100 peer-reviewed papers. His current interests are in the field of Brain-Computer Interfaces, real-time data processing of EEG and MEG signals, and modeling and engineering aspects related to physiological data acquisition and processing. He has also developed several commercial and free software tools in the bioengineering research field, which include the first multiplatform BCI framework and the first commercial long-term VideoEEG software acquisition and analysis tool in the world.

Milan Djilas obtained a Dipl. Ing. degree in Electrical Engineering from the University of Belgrade in Serbia, a M.Sc. degree in Biomedical Engineering from the University of Aalborg in Denmark, and a Ph.D. degree in Neural Prosthetics from the University of Montpellier II in France. The topic of his Ph.D. was using implantable neural prosthetics for restoring limb movement in individuals with paralyzed limbs. He was a postdoctoral research fellow at the Vision Institute in Paris, working with Dr. Picaud on the development of high-resolution retinal prosthetics to restore vision to the blind. He is currently an advanced consultant in Altran Switzerland, consulting other companies in the life sciences and healthcare industries.

Joanna Dreszer Drogorób is teaching and research assistant at Department of Cognitive Science and Epistemology, Nicolaus Copernicus University in Torun, and researcher at Center for Modern Interdiscyplinary Technologies, Nicolaus Copernicus University in Toruń. She has a Masters degree and a doctorate in psychology. She is involved as researcher in several scientific projects concerning cognitive science and developmental psychology. She also teaches courses on neuropsychology, neurorehabilitation, and neuroimaging techniques at Nicolaus Copernicus University, Poland. Her scientific interests focus on developmental processes and disorders, time perception, and neuroimaging of sensory and cognitive processes. 
Thomas Emmerling was born on September 23, 1986 in Haan (Rheinl.), Germany. In 2005, he completed his secondary school education at the Ludwig-Meyn-Schule in Uetersen. In 2006, he studied Psychology at the University of Trier, Germany, and worked there as a research assistant in the psychophysiological laboratory under the supervision of Dr. Ewald Naumann. In 2010, he completed a one-year internship at the Department of Cognitive Neuroscience at Maastricht University (The Netherlands), where he conducted empirical work for his master thesis about hybrid brain-computer interfaces integrating electroencephalography and functional near-infrared spectroscopy under the supervision of Prof. Dr. Rainer Goebel and Dr. Bettina Sorger. After obtaining his master's degree ("Diploma”) in 2011, he now works as a PhD candidate on high-field fMRI brain-computer interfaces at the Department of Cognitive Neuroscience at Maastricht University.

Helena Erlbeck holds a Master's degree in psychology after studying at the Universities of Chemnitz in Germany. She is currently doing her PhD at the University of Würzburg (Germany). Her research interests involve information processing in patients with disorders of consciousness and neurophysiological correlates of attention. Furthermore, she is part of an international project, where she is working on a neurofeedback-based rehabilitation program for stroke patients.

Rita Formisano, Director of Post-Coma Unit (Santa Lucia Foundation, Rehabilitation Hospital and Research Institute in Rome). She is author of 118 peer-reviewed papers on international journals, and reviewer for 5 of these. She is also engaged in translational clinical research of severe brain injury patients with Disturbance Of Consciousness (DOC) for 25 years. She was research fellow at the University Neurology of Innsbruck (Austria) from 1984 to 1987, recognized by Italian Health Ministry (03/10/1994). She is involved in national and international protocols (NIH project on biomarkers in severe brain injury), multicentric studies (QOLIBRI), and National Consensus Conferences on severe brain injury. She is also member of national and International Societies on Neurorehabilitation and DOC.

Alex Gillespie is Associate Professor in Social Psychology at the London School of Economics, and Editor of Journal for the Theory of Social Behaviour. He has a BA in Psychology from Trinity College Dublin, an MSc in Social Psychology from the LSE, and a PhD from the University of Cambridge. His theoretical work focuses on joint activity, scaffolding, and perspective taking. His empirical research examines formal and informal care interactions and how these can be simulated using assistive technology for cognition. He thinks that we are living through a digital revolution, which will transform society and be recognised by future historians as equivalent in significance to the industrial revolution. 
Rainer Goebel (1964), professor of Cognitive Neuroscience, studied psychology and computer science in Marburg (Germany, 1983-1988). He received his PhD at the Technical University of Braunschweig (Germany, 1994). From 1995-1999, he was Postdoc at the Max Planck Institute for Brain Research in Frankfurt/Main (Germany). In 2000, he was appointed full professor at Maastricht University (Faculty of Psychology and Neuroscience, The Netherlands). Since 2005, he has been director of the Maastricht Brain Imaging Centre. From 2006-2009, he was chair of the Organization for Human Brain Mapping. Since 2008, he has been team leader of the neuromodeling and neuroimaging group at the Netherlands Institute for Neuroscience of the Royal Netherlands Academy of Arts and Sciences. His research includes high-resolution functional imaging of the visual system, artificial large-scale columnar-level neural network models of visual cortex, as well as development of hemodynamic brain-computer interfaces (including neurofeedback) and of new analysis methods for neuroimaging data.

Christoph Guger studied biomedical engineering at the University of Technology Graz, Austria. He pursued his $\mathrm{PhD}$ in the area of real-time analysis of EEG data for the realization of a brain-computer interface. Since 1999, Christoph Guger works for Guger Technologies OEG and g.tec medical engineering Gmbh, both based in Austria. The companies develop hardware and software for biosignal acquisition and analysis. Christoph Guger is partner in several EU-funded projects, like Presenccia, ReNaChip, synthetic forager, SM4All, Decoder, and Brainable, etc.

Jufang He obtained a B.Eng. and M.Eng. degree in Engineering at the Harbin Institute of Technology, a Doctoral Degree in Medical Science at the University of Tokushima, and a second Doctoral Degree in Engineering at the University of Tokyo, Japan. Before joining the City University of Hong Kong in September 2013, Prof. He had worded at Riken (Japan), University of Tokushima, and Advanced Research Laboratory of Hitachi Ltd. for 5 years, and The Hong Kong Polytechnic University (PolyU) for 15 years. Prof. He combines electrophysiological, anatomical, and engineering approaches to answer fundamental questions of hearing, and learning and memory. Besides Neurobiology, Prof. He has developed an electronic "bat ear" for people with visual impairments and a "motolink" for bypassing the spinal cord injury. Prof. He is directing a collaborative team between CAS and CityU for developing a memory drug.

Lizette Heine (1988) is a PhD student at the coma science group at the University of Liege (Belgium). In 2010, she finished her bachelor in biological psychology at Maastricht University (The Netherlands). During her master in Neuropsychology at the same institution, she focused under the supervision of Dr. Bettina Sorger and Prof. Dr. Rainer Goebel on resting state and active imagery paradigms using Functional Magnetic Resonance Imaging (fMRI) and Functional Near-Infrared Spectroscopy (fNIRS). Since October 2012, she has worked as a research fellow in biomedical sciences at the coma science group under the supervision of Prof. Dr. Steven Laureys. Her work mainly focuses on assessment of residual cognitive processing in non-communicating patients through the combination of fMRI, diffusion-weighted imaging, and clinical neuropsychological testing. 
Petar Horki received the BSc and MSc degree in telematics from the Graz University of Technology, Austria, where he is currently pursuing his $\mathrm{PhD}$ degree. He is member of the Laboratory for BrainComputer Interfaces (BCI-Lab) at Graz University of Technology since 2009. His research interests include direct brain-computer interfacing based on EEG signals and biosignal processing.

Wei-Yen Hsu received the M.S. and Ph.D. degrees in the Department of Computer Science and Information Engineering from National Cheng Kung University, Tainan, in 2001 and 2008, respectively. $\mathrm{He}$ is an assistant professor in the Department of Information Management, National Chung Cheng University. His research interests include medical image processing, biomedical signal processing, and neuroscience methods.

Silvio Ionta received the MSc in Experimental Psychology at the University of Rome "Sapienza" (Italy) working in the Social and Cognitive Neuroscience Laboratory. For his PhD in Functional Neuroimaging, he moved to the Institute of Advanced Bio-Medical Technologies at the Dept. of Clinical Sciences and BioImaging, University of Pescara-Chieti "G. D'Annunzio" (Italy). Granted by the Italian Ministry of Instruction, he completed the PhD studentship at the Laboratory of Cognitive Neuroscience at the Ecole Polytechnique Fédérale de Lausanne (Switzerland), where he also worked as post-doctoral researcher. Supported by the program NCCR Neural Plasticity and Repair of the Swiss Nation Science Foundation, he started his research assistant position at the Rehabilitation Engineering Lab at the Eidgenössische Technische Hochschule Zürich (Switzerland). He is currently lecturer at the Department of Radiology, Centre Hospitalier Universitaire Vaudois and University of Lausanne (Switzerland).

Tomasz Komendziński is senior lecturer at Department of Cognitive Science and Epistemology, Nicolaus Copernicus University in Toruń, and researcher at Center for Modern Interdisciplinary Technologies, Nicolaus Copernicus University in Torun. He is the initiator, co-creator, and coordinator of InteRDoCTor research team, well known from many previous research projects and conferences. He has a Masters degree and a Doctorate in Philosophy. He is involved as researcher in several scientific projects concerning philosophy and cognitive science. He also teaches courses on philosophy and cognitive science at Nicolaus Copernicus University, Poland. His scientific interests focus on human perception, sensory and cognitive processes.

Boris Kotchoubey finished 1st Moscow Medical Institute (General Medicine) and the Russian Academy for Educational Sciences (Psychology) with a Ph.D in Psychology. Presently, he is professor ("Akademischer Rat") at the University of Tübingen. His central research question is, What are the natural (biological, physiological) underpinnings of specific human qualities such as consciousness, language, and freedom? Research activities include electrocortical processes and behavioural control, learning processes in the development and treatment of neurological diseases, biological and neurophysiological basis of consciousness and freedom, altered states of consciousness, and disorders of consciousness. 
Andrea Kübler is Professor of Psychological Intervention at the University of Würzburg. She received Master Degrees in Biology and Psychology and her PhD in Biology. During her PhD, she developed a BCI on the basis of slow cortical potentials for communication with locked-in patients. She is an expert in Neurofeedback and training locked-in patients with different BCI. Currently, she is investigating how affective states and motivation influences BCI performance, what predicts BCI performance, and how BCI can be brought to the patients' bedside. She is further investigating how BCIs can be used for the detection of consciousness in non-responsive patients and for cognitive rehabilitation after stroke. Other research interests are addiction and eating disorders, quality of life and coping with disease, and intervention for children and adolescents with sleeping disorders. She has numerous publications in the $\mathrm{BCI}$ field and holds four national and international grants for research on BCI.

Steven Laureys, MD, PhD, is director of the Coma Science Group at the Neurology Department and Cyclotron Research Centre of the University Hospital and University of Liège, Belgium. He is clinical professor of neurology, board-certified in neurology and in palliative medicine, and research director at the Belgian National Fund for Scientific Research. His team studies the neural basis of human consciousness (coma, anesthesia, hypnosis, and sleep). He assesses the recovery of neurological disability and neuronal plasticity in acquired brain injury (e.g., comatose, "vegetative"/unresponsive, minimally conscious, and locked-in syndromes) confronting clinical expertise and behavioral evaluation with multimodal neuroimaging (positron emission tomography and magnetic resonance imaging) and electrophysiology studies (electroencephalography coupled to transcranial magnetic stimulation), and also deals with the ethical implications of this translational clinical research. He is chair of the World Federation of Neurology Applied Research Group on Coma and the European Academy of Neurology Subcommittee on Disorders of Consciousness.

Heung-No Lee (S'94-M'99) received the B.S., M.S., and Ph.D. degrees in electrical engineering from the University of California, Los Angeles (UCLA), in 1993, 1994, and 1999, respectively. From March 1999 to December 2001, he was with the Network Analysis and Systems Department, Information Science Laboratory, Hughes Research Laboratories, Malibu, CA, where he led a number of research projects as the Principal Investigator including traffic modeling for tactical Internet (under the Defense Advanced Research Projects Agency [DARPA] Advanced Technology Office [ATO] Adaptive Signal Processing and Networks [ASPEN] Program), future tactical networking system, capacity analysis for satellite networks using realistic input traffic, and broadband wireless modem. In 2002, he joined the Department of Electrical Engineering, University of Pittsburgh, PA. Since January 2009, he has been an Associate Professor in the Department of Information and Communications, Gwangju Institute of Science and Technology, Korea. His current research interests include information and signal processing theories for wireless network and biomedical applications.

Seungchan Lee received his undergraduate degree in Electrical Engineering from Chungbuk National University, South Korea, in 2009 and M.S. in Information and Communications from Gwangu Institute of Science and Technology (GIST), South Korea, in 2012. He is currently a Ph.D. student at the INFONET Lab in GIST of Prof. Heung-No Lee. His research interests include brain-computer interfaces, neuro-rehabilitation, BCI system, human motor control, and bio-signal processing. 
Damien Lesenfants received the Biomedical Engineering degree from the Université de Liège (ULg, Liège, Belgium) in June 2010. During his master thesis, he worked on MIVIP and OPTIVIP European Projects in collaboration with the University of Louvain (UCL, Belgium) on the improvement of a visual prosthesis for blind patients. He currently makes his Ph.D. under the supervision of Professor $\mathrm{S}$. Laureys and Dr Ir Quentin Noirhomme at the Coma Science Group, Cyclotron Research Centre (ULg). His studies focus on (1) finding new diagnosis and prognosis measures based on functional neuroimaging (mainly EMG, EEG, and BCI) for patients with disorders of consciousness and (2) developing new communication tool for brain-injured patients.

Monika Lewandowska is a research assistant at the Bioimaging Research Center of the Institute of Physiology and Pathology of Hearing, Poland. She has a Masters degree in Psychology (specialty: clinical neuropsychology) at the Warsaw University and a Doctorate in Biological Sciences from the Nencki Institute of Experimental Biology, Poland. She is currently principal researcher for several project aimed at developing new methods for neurorehabilitation and application of the EEG and fMRI and EEG/fMRI to investigate neuroplasticity of the human brain. She also teaches courses on neuropsychology, neurorehabilitation, and neuroimaging techniques at Nicolaus Copernicus University, Poland. Her scientific interests focus on central auditory processing disorders, tinnitus, time perception, and neuroimaging of sensory and cognitive processes.

Zulay Lugo, Specialist in Psychiatry (1996) and Specialist in Neurology (2003), received her diplomas at the Universidad Central de Venezuela (Caracas, Venezuela). After a long clinical practice, including two years of internship at the Centre Hospitalier Sainte-Anne and the Centre Hospitalier de Meaux in France, with Attestations of in-depth training in Psychiatry and Neurology delivered by the Universities Paris XI (2007) and Paris VII (2008), respectively, she started a researcher career in 2008, getting in 2010 a Master in Cognitive Sciences at the Ecole Normale Supérieure de Paris (jointly with the University Paris V and the Ecole des Hautes Etudes en Sciences Sociales). She is currently PhD candidate at the University of Liège (Belgium) and the University of Wurzburg (Germany) working on brain-computer interfaces and disorders of consciousness. She participated at the DECODER project working with patients from the French Association for the Locked-In Syndrome (ALIS).

Donatella Mattia received her MD degree at University of Rome "Sapienza," Italy in 1987. In 1991, she became neurologist at the same university. She received the $\mathrm{PhD}$ degree in "Physiopathology of Movement Disorders" in 1996, from the Department of Neuroscience, "Sapienza" University of Rome. During her PhD program, she was a research fellow at the Montreal Neurological Institute and Department of Neurology and Neurosurgery, McGill University, Montréal, Québec, Canada. She started in 1998 her research activity at the Neurophysiology Dept. at the Fondazione Santa Lucia, IRCCS, Rome, Italy, and in 2004, she became the director of the Laboratory of Neuroelectrical Imaging and Brain Computer Interface. Research interests are focused on advanced EEG signal processing to investigate the basis of human cognitive and motor function and application of the EEG-based BCI technology in a clinical setting as rehabilitation tool. 
Emilia Mikołajewska, $\mathrm{PhD}$, is physiotherapist and scientist, senior assistant in Rehabilitation Clinic, Military Clinical Hospital No. 10 with Polyclinic in Bydgoszcz, member of the InternationalInterdisciplinary Research on Disorders for Consciousnes in Torun (InteRDoCTor) research team. She is author of 8 books, 40 book chapters, and 200 articles in the area of rehabilitation and physiotherapy (especially neurorehabilitation and neurological physiotherapy). She is a reviewer in 18 scientific journals (also member of 3 editorial boards) and several book chapters. She also teaches courses on neuro rehabilitation and assistive technology and acts as supervisor of three specialization candidates. Her research interests cover: neurorehabilitation of adult patients, including post-stroke; neurorehabilitation of children; increasing of patients' quality of life using assistive devices, telemedicine, tele-rehabilitation, cyber therapy, rehabilitative robotics, applications of IT, AI, and integrated systems in medicine; cooperation within therapeutic multidisciplinary team; evidence based medicine in clinical practice; and biomedical engineering in rehabilitation.

Dariusz Mikołajewski is Polish engineer and scientist, specialist in IT, applications of IT in medical sciences, biocybernetics, and medical robotics. He works for three research centers: 1) Institute of Mechanics and Applied Computer Science, Kazimierz Wielki University in Bydgoszcz, 2) Department of Informatics, Nicolaus Copernicus University in Toruń, 3) Centre for Modern Interdisciplinary Technologies, Nicolaus Copernicus University in Torun. He is also member of the InteRDoCTor research team and author of approximately 100 articles in the area of medical IT, biocybernetics, and biomedical engineering.

Martin M. Monti, Ph.D., is Assistant Professor at the Cognitive Psychology of Princeton University. His research focuses on two of the most fundamental aspects of being human: (1) the interplay between language and thought and (2) consciousness and cognition in coma, vegetative, and minimally conscious state.

Gernot Müller-Putz is Head of the Institute for Knowledge Discovery, Graz University of Technology, Austria. He is also Head of the Laboratory for Brain-Computer Interfaces (BCI Lab) at Graz University of Technology. In 2004, he received his PhD in Electrical Engineering ("New Concepts in Brain-Computer Communication Use of Steady-State Somatosensory Evoked Potentials: User Training by Telesupport and Control of Functional Electrical Stimulation") from Graz University of Technology, where, beginning in 2000, he worked on non-invasive Electroencephalogram-Based (EEG) BrainComputer Interfacing (BCI) for the control of neuroprosthetic devices. In 2008, he received his "venia docendi" for medical informatics ("Towards EEG-Based Control of Neuroprosthetic Devices") at the faculty of computer science at Graz University of Technology. His research interest includes EEG-based neuroprosthesis control, hybrid BCI systems, the human somatosensory system and assistive technology. 
Lorina Naci is a Postdoctoral Fellow at the Brain and Mind Institute, Western University, in Ontario, Canada. Dr. Naci's work uses functional neuroimaging (EEG, fMRI) to develop novel techniques that probe residual cognition, conscious awareness, and communication in patients with disorders of consciousness. These involve active and passive paradigms aimed at mapping out sensory (audition, vision) as well as higher-order cognitive functions, such as selective attention. Her work has been covered extensively in the international media and published in several peer-reviewed clinical and basic science journals, including JAMA Neurology, Journal of Neuroscience, Annals of Neurology, Schizophrenia Research, Frontiers in Integrative Neuroscience, Human Brain Mapping, etc.

Quentin Noirhomme is working at the Coma Science Group of University of Liège. His researches focus on finding new diagnosis and prognosis measures-based functional neuroimaging (mainly EEG, fMRI) for patients with disorders of consciousness, studying change in functional and effective connectivity and developing Brain-Computer Interface (BCI) for detecting response to command and communication in disabled patients.

Brian O'Neill is a Consultant in Neuropsychology and Rehabilitation with the Brain Injury Rehabilitation Trust, Glasgow, and Research Fellow at the University of Stirling. He has a BA in Psychology from Trinity College Dublin, a Doctorate in Clinical Psychology, and a MSc in Clinical Neuropsychology from the University of Glasgow. Since qualification, he has worked with people requiring rehabilitation after illnesses and accidents. He has worked with a variety of client groups across health settings. He sees rehabilitation as enabling people to participate in meaningful social lives. Technologies are tools with the potential to aid that process by enabling activity and participation. His other interests include the prediction of rehabilitation outcome and the use of assistive technology for cognition to improve functional ability.

Adrian M. Owen is currently the Canada Excellence Research Chair(CERC) in Cognitive Neuroscience and Imaging at the University of Western Ontario, Canada. His work combines structural and functional neuroimaging with neuropsychological studies of brain-injured patients. His most recent work, reported in the journals Science (2006), The New England Journal of Medicine (2010), and The Lancet (2011) has shown that functional neuroimaging can reveal conscious awareness in some patients who appear to be entirely vegetative, and can even allow some of these individuals to communicate their thoughts and wishes to the outside world. These findings have attracted widespread media attention on TV, radio, in print and online, and have been the subject of several TV and radio documentaries. Dr. Owen has played multiple editorial roles including 8 years as Deputy Editor of The European Journal of Neuroscience. Since 1990, Dr. Owen has published over 200 articles and chapters in scientific journals and books. 


\begin{abstract}
Alessandra Pedrocchi received the M.S. degree in Electrical Engineering and the Ph.D. degree in Bioengineering from the Politecnico di Milano in 1997 and 2001, respectively. Since June 2008, she is Assistant Professor in tenure track at the Department of Electronics Informatics and Bioengineering of the Politecnico di Milano, where she teaches Neuroengineering in the Master of Science in Biomedical Engineering. Her research activities are carried out at the NearLab in the field of biomechanics in motor control, neuroengineering, and neurorehabilitation (www.nearlab.polimi.it). She has been the Project Manager of the MUNDUS project (FP7 ICT-2009.7.2), and she is the Politecnico PI for REALNET project (FP7 Obj ICT-2009.6).
\end{abstract}

Frédéric Pellas, MD, is neurologist and is the director of post-coma unit of the neurorehabilitation at the University Hospital of Nîlmes (Fr). He works currently with non-responsive patients (coma arousal, VS, MCS, LIS, akinetic mutism) since 1995. In this field, he is the coordinator of a National University Course on TBI patients and has published three books (Editor in Chief) on TBI, coma, VS, and LIS medical and rehabilitative management. He organised several national conferences on TBI, LIS, PVS, and MCS (Montpellier 2007, 2008, 2009). His medical interest in LIS began in 1998 when he joined the Locked-In Syndrome Association (ALIS). He co-organised with them many congresses and post-graduate courses on LIS care. He is co-author of 13 papers published on peer-reviewed journals ( 5 of them on LIS with Steven Laureys and ALIS), 10 book chapters, and 7 books as editor. He has been invited as an expert on Brain-Computer Interfaces meeting in Paris (November 4th and 5th, 2009) funded by the IRME (Institut Recherche Moelle et Encéphale), the FRM (Fondation Recherche Médicale), and ICM (Institut du Cerveau et de la Moelle Epinière). He participated as an expert in national boards (AFSSAPS Spasticity treatments national guidelines, SOFMER board and conference on referral of post-stroke patients). He is associated research member since 2009 of the Research Unit EA 2991 "Efficience \& Déficience Motrices" (EDM) at the University of Montpellier I (Director Denis Mottet).

Serge Picaud is currently heading the team for retinal information processing at the Vision Institute in Paris. The team has investigated the physiology and pharmacology of retinal cells in normal and pathological conditions. It recently focused on developing strategies for restoring vision in blind patients having lost their photoreceptors. The first approach aims at generating high-resolution retinal prostheses using new 3D implant designs, new materials such as diamond or graphène. In addition, new visual information encoding systems are generated to mimic the high retinal dynamic. Finally, reactivation of residual neurons is attempted with optogenetic tools (photosensitive proteins producing currents). In collaboration with Dr. Roska, reactivation of residual non-photosensitive cone photoreceptors was, for instance, achieved in blind mice and in the postmortem human retina. The two spinoff companies, Pixium Vision and Gensight, were created to bring prostheses and optogenetic therapy to clinical trials.

Iolanda Pisotta, psychologist, received her MSc in "Diagnosis and Rehabilitation of Cognitive Disorders" at the University of Rome "Sapienza" (Italy), working in the Ataxia lab at Santa Lucia Foundation. After obtaining a research assistant fellowship at the Santa Lucia Foundation, where she worked in the "Clinical Movement Analisys and Research Lab," she started her PhD studentship in Neuroscience at University of Rome "Sapienza." Supported by the "TOBI," "Better," and "MINDWALKER" projects, funded by the EU, 7th Framework Program, she is currently involved in research activities in the field of non-invasive Brain-Computer Interfaces and neuro-rehabilitation robotics. 
Christoph Pokorny received the B.Sc. and M.Sc. degrees in Telematics in 2006 and 2009, respectively, from the Graz University of Technology, Graz, Austria, where he is currently working toward the Ph.D. degree at the Institute for Knowledge Discovery. His research interests include signal processing, electrical engineering, and Brain-Computer Interfaces (BCIs) for non-responsive patients.

Lucia Rita Quitadamo is a biomedical engineer and currently a post-doctoral researcher at the University of Tor Vergata, Rome, Italy. She works with Brain-Computer Interface (BCI) technologies, particularly focusing on the detection and classification of mental states in non-responsive/noncommunicative patients and on the implementation of metrics for the evaluation and standardization of BCI systems performances. She also studies the development and the implementation of advanced tools and methodologies for biomedical signals (EEG, MEG, EMG) processing for Human-Computer Interaction purposes. She worked in different European projects and developed different international research collaborations. She is author and coauthor of numerous international journals papers and conference proceedings.

Ruben Real holds a diploma in psychology after studying at the Universities of Aberdeen (UK) and Göttingen (Germany). He is doing a PhD at the University of Würzburg (Germany) and has won a scholarship of the Würzburg research training group emotions. His research interests involve information processing in disorders of consciousness and the quality of life of patients with severe diseases. He is part of CONTRAST, an international research project concerning neurofeedback-based rehabilitation for stroke patients.

Monica Risetti received her Psychology degree at University of Rome "Sapienza," Italy in 2003. She attended the Specialization School at the same University and became Neuropsychologist in 2010. In 2009, she started her research activity at the Laboratory of Neuroelectrical Imaging and Brain-Computer Interface at the Fondazione Santa Lucia, IRCCS, Rome, Italy. Her research interests are focused on EEG-based BCI technology in a clinical setting as a tool directed at assisting, augmenting, or repairing human cognitive or sensory-motor functions.

Martin Rohm received the Dipl.-Ing. Degree in Electrical Engineering from the Technical University of Darmstadt, Germany, in 2008. He is currently employed in the research group "Experimental Neurorehabilitation" (Dr.-Ing. R. Rupp) of the Spinal Cord Injury Center (Prof. N. Weidner) of Heidelberg University Hospital, Germany. His main research interests are in the field of rehabilitation engineering for individuals with spinal cord injury. This includes Functional Electrical Stimulation mainly of the upper extremity, application of Brain-Computer Interfaces and the use of impedance tomography for determining the human bladder volume. He is author of more than 25 journal and conference contributions. 
Rüdiger Rupp received the Dipl.-Ing. Degree in Electrical Engineering and his Ph.D. degree from the Technical University of Karlsruhe, Germany, in 1994 and 2008, respectively. After working at the Institute for Biomedical Engineering and Biocybernetics (Prof. G. Vossius) until 1996, he is currently the head of the research group "Experimental Neurorehabilitation" of the Spinal Cord Injury Center (Prof. N. Weidner) of Heidelberg University Hospital, Germany. His main research interests are in the field of rehabilitation engineering for individuals with spinal cord injury. This includes neuroprosthetics mainly of the upper extremity, functional electrical stimulation for therapeutic purposes, development and clinical validation of novel methods and devices for locomotion therapy, and standardized outcome documentation including gait analysis. He is author of more than 180 journal, book, and conference contributions and holds two patents. His work has been awarded several times and he is member of IEEE, IFESS, VDE, DGOOC, ASIA, and ISCoS.

Matthias Schneiders received the B.Sc. degree in medical informatics from the University of Heidelberg and University for applied Science of Heilbronn, Germany, in 2010. He is currently employed in the research group "Experimental Neurorehabilitation" (Dr.-Ing. R. Rupp) of the Spinal Cord Injury Center (Prof. N. Weidner) of Heidelberg University Hospital, Germany. His main research interests are in the field of rehabilitation engineering for individuals with spinal cord injury. This includes Functional Electrical Stimulation mainly of the upper extremity, application and development of Brain-Computer Interfaces, and the use of sensors for motion analysis.

Younghak Shin received his undergraduate degree in Electronics and Communications from Kwangwoon University, South Korea, in 2009 and M.S. in Information and Communications from Gwangu Institute of Science and Technology (GIST), South Korea, in 2011. He is currently a Ph.D. student at the INFONET Lab in GIST of Prof. Heung-No Lee. His research interests include brain-computer interfaces, neuro-rehabilitation, BCI system, human motor control and bio-signal processing.

Bettina Sorger is an assistant professor at the Department of Cognitive Neuroscience of the Faculty of Psychology and Neuroscience at Maastricht University (The Netherlands). She studied psychology at Cologne University (Germany). After she obtained her Master's degree ("Diplom") in 2002, she worked at the University Hospital of Cologne advancing clinical (including intra-operative) applications of Functional Magnetic Resonance Imaging (fMRI). From 2005 to 2009, she worked on her PhD project on the development of Brain-Computer Interfaces (BCIs) based on real-time fMRI at Maastricht University. After her promotion in 2010, she joined the Coma Science Group at the University of Liège (Belgium) applying fMRI-based communication BCIs in "locked-in" patients. She returned to Maastricht University in 2011 and is currently mainly working on transferring the developed alternative communication methods to mobile Functional Near-Infrared Spectroscopy (fNIRS). Her research activities focus on the development of clinical BCIs for brain-based communication and neurofeedback therapy. 
Jlenia Toppi received her master degree in Biomedical Engineering at University of Rome "Sapienza," Italy in 2009. She received the PhD degree in "Biomedical Engineering" from the Department of Computer, Control, and Management Engineering "Antonio Ruberti," University of Rome "Sapienza," Italy in 2013. In July 2013, she got a post-doc position at the Department of Computer, Control, and Management Engineering “Antonio Ruberti," University of Rome "Sapienza," Italy. Since 2009, she conducted her research activity at the Neuroelectrical Imaging and Brain Computer Interface Laboratory at the Fondazione Santa Lucia, IRCCS, Rome, Italy. Research interests are focused on the development of methodologies for the study of cerebral activity and connectivity from non-invasive EEG recordings, for clinical and rehabilitative purposes.

Sandra Veser studied Psychology at the University of Landau and Dresden. In 2011, she finished her Ph.D. in Psychology at the University of Leipzig. Presently, she is post-doc at the Institute of Medical Psychology at the University of Tübingen. Her research interests are disorders of consciousness with the main focus on neurophysiological diagnosis and communication, visual attention effects especially neurophysiological similarities, and differences of voluntary and involuntary attention, visual awareness, and binocular rivalry.

Tomasz Wolak is a head of the Bioimaging Research Center and Senior Research Fellow at the World Hearing Center of Institute of Physiology and Pathology of Hearing in Kajetany, Poland. He has a Masters degree and a doctorate at Medical and Nuclear Electronics Division of the Warsaw University of Technology. He actively participated in numerous research projects on application of an fMRI technique for studying sensory and cognitive processes in humans. He was involved in the formation of the Laboratory of Diagnostic Imaging of the Institute of Physiology and Pathology of Hearing. In March 2009, the Laboratory has evolved into the Bioimaging Research Center, equipped with modern 3Tmagnetic resonance scanner. His main interests are functional magnetic resonance imaging, brain image segmentation, image analysis and visualization, language and auditory functional studies. He collaborated with many clinical and scientific centers in Poland, where he introduced the technique of fMRI into clinical practice. He has13 years of experience in the subject of neuroimaging, more than 2,000 performed studies and analyses, and participated in several research projects related to the fMRI and EEG/fMRI.

Soogil Woo received his undergraduate degree in Electronics Engineering from Jeonbuk National University, South Korea, in 2012. He is currently a M.S. student at the INFONET Lab in GIST of Prof. Heung-No Lee. His research interests include brain-computer interfaces, neuro-rehabilitation, BCI application, BCI system, and bio-signal processing. 


\section{Chapter 10 \\ Brain-Computer Interfaces for Assessment and Communication in Disorders of Consciousness}

\author{
Christoph Guger \\ Guger Technologies, Austria \\ Bettina Sorger \\ Maastricht University, The Netherlands \\ Quentin Noirhomme \\ University of Liege, Belgium \\ Lorina Naci \\ University of Western Ontario, Canada \\ Martin M. Monti \\ University of California, Los Angeles, USA \\ Ruben Real \\ Universität Würzburg, Germany \\ Christoph Pokorny \\ Graz University of Technology, Austria \\ Sandra Veser \\ University of Tübingen, Germany \\ Zulay Lugo \\ University of Liège, Belgium \\ Lucia Quitadamo \\ University of Tor Vergata, Italy
}

\author{
Damien Lesenfants \\ Université de Liège, Belgium \\ Monica Risetti \\ Fondazione Santa Lucia, Italy
}

Rita Formisano

Ospedale di Riabilitazione Fondazione Santa Lucia, Italy

Jlenia Toppi

University of Rome "La Sapienza," Italy

Laura Astolfi

Università di Roma “La Sapienza," Italy

Thomas Emmerling

Maastricht University, The Netherlands

\section{Lizette Heine}

Maastricht University, The Netherlands

Helena Erlbeck

Universität Würzburg, Germany

Petar Horki

Graz University of Technology, Austria

Boris Kotchoubey

University of Tübingen, Germany 
Luigi Bianchi

"Tor Vergata" University of Rome, Italy

Donatella Mattia

Fondazione Santa Lucia, Italy

Rainer Goebel

Maastricht University, The Netherlands

Adrian M. Owen

Western University, Canada
Frederic Pellas

Association Locked-In Syndrome, France

Gernot Müller-Putz

Graz University of Technology, Austria

Steven Laureys

University of Liege, Belgium

Andrea Kübler

Universität Würzburg, Germany

\begin{abstract}
Many patients with Disorders of Consciousness (DOC) are misdiagnosed for a variety of reasons. These patients typically cannot communicate. Because such patients are not provided with the needed tools, one of their basic human needs remains unsatisfied, leaving them truly locked in to their bodies. This chapter first reviews current methods and problems of diagnoses and assistive technology for communication, supporting the view that advances in both respects are needed for patients with DOC. The authors also discuss possible solutions to these problems and introduce emerging developments based on EEG (Electroencephalography), fMRI (Functional Magnetic Resonance Imaging), and fNIRS (Functional Near-Infrared Spectroscopy) that have been validated with patients and healthy volunteers.
\end{abstract}

\section{INTRODUCTION}

The clinical care and rehabilitation approaches involving patients diagnosed with disorders of consciousness (DOC) are of great medical and social importance. The progressive increase in the number of surviving patients and their increasing life expectancies, in all industrialized countries, reflects the advancement of knowledge and techniques in the field of reanimation, as well as improved quality of care. An accurate early diagnosis is indispensable to develop early and effective standards of care, appropriate to an individual patient's condition. The clinical diagnosis of these patients is a major challenge because of the very fine line between the Vegetative State/ Unresponsive Wakefulness Syndrome(VS/UWS; Laureys et al., 2010), characterized by preserved arousal in the absence of behavioral signs of awareness, the Minimally Conscious State(MCS), with non-reflexive albeit inconstant purposeful behaviors, and the Locked-In Syndrome (LIS), in which consciousness is fully preserved (Giacino et al., 2002). Recent work identified two groups within this patient population - those who show higher-order signs of consciousness (e.g., command following, intelligible verbalization, and non functional communication; i.e. MCS plus) versus those who show only low-level signs of consciousness (e.g., visual pursuit of a salient stimulus, noxious stimulation localization, appropriate emotional response; i.e. MCS minus) (Bruno et al., 2011; Bodart et al., 2013).

Despite the efforts already made to improve the instrumental diagnosis, differential diagnosis between different patients with an altered state of consciousness is eminently clinical and based on a list of items that the patient is unable to perform. The LIS is a term introduced by Plum and Posner to describe a neurological condition 
of quadriplegia and anarthria associated with ventral pons infarction (Plum \& Posner, 1966). The American Congress of Rehabilitation Medicine defined the LIS as: i) the presence of sustained eye opening, ii) the preservation of cognitive skills, iii) a severe hoarseness or hypophonia iv) quadriplegia or quadriparesis, and v) a primary mode of communication using eye movements or blinking (American Congress of Rehabilitation Medicine, 1995). The main etiology of LIS is stroke (Patterson \& Grabois, 1986). The diagnosis of this condition is particularly challenging because patients cannot communicate by the usual means. These patients are fully conscious but completely or almost completely unable to move and speak, so they can easily be mistaken for VS patients.

Evidence-based medicine (EBM) has been defined as the integration of current best evidence with clinical experience and patients' values and preferences in clinical decision-making process (Brownson et al., 2012). With the introduction of EBM, the clinicians are exhorted to consider all three factors in the process to foster the evidencebased-decision-making (EBDM) in public health. These concepts appear of utmost relevance when dealing with disorders of consciousness characterizations, which entail a broad spectrum of entities that are often misdiagnosed.

In this context, the development of braincomputer interface (BCI) tools, as well as other paraclinical instruments and methods, can facilitate the differentiation of these clinical states. BCIs are devices that allow users to communicate directly by means of brain activity. Recently, BCIs have become much more practical and flexible, due to advances such as dry electrodes, high-impedance amplifiers, better interfaces (Kaufmann et al., 2013a), and improved testing with patients in clinical settings (Zickler et al., 2011). Hence, BCIs are becoming useful to broader groups (Guger et al., 2012; Wolpaw and Wolpaw, 2012; Allison et al., 2013).
The main objective of this chapter is to show how BCI technology is becoming useful to DOC patients. The two main functions are for communication, which has been well established for BCIs, and detection of consciousness, which is relatively unexplored. We will first review background material showing that conventional methods need improvement, and then introduce EEG methods. This sets the stage to present new research studies through the European Commission (EC) research project DECODER (www.decoderproject.eu), using EEG, fMRI and fNIRS to show that BCI technology can supplement conventional methods.

\section{BACKGROUND}

Forty-three percent of patients diagnosed as being in the VS are reclassified as (at least) minimally conscious when assessed by expert teams (Schnakers et al., 2009). OtherDOC patients might appear non-communicative through standard clinical testing, but could be able to communicate with newer methods. Furthermore, some DOC patients have transient periods of relative wakefulness, but remain unaware of their surroundings. The European project DECODER (see Figure 1) aimed at using BCI technology to identify DOC patients who might be able to communicate through newer, BCI based technologies.

One recent study showed that patients identified as being in a vegetative state VS were not only consciously aware, but could answer yes or no questions (Monti et al., 2010). This work demonstrates that some patients meet all behavioural criteria for VS, but nevertheless have covert awareness. Some research to assess cognitive activity in DOC patients relies on fMRI. Functional MRI can be very powerful but also entails major limitations, as discussed in the next section. 
Figure 1. A graphical depiction of how the DECODER project could facilitate DOC assessment
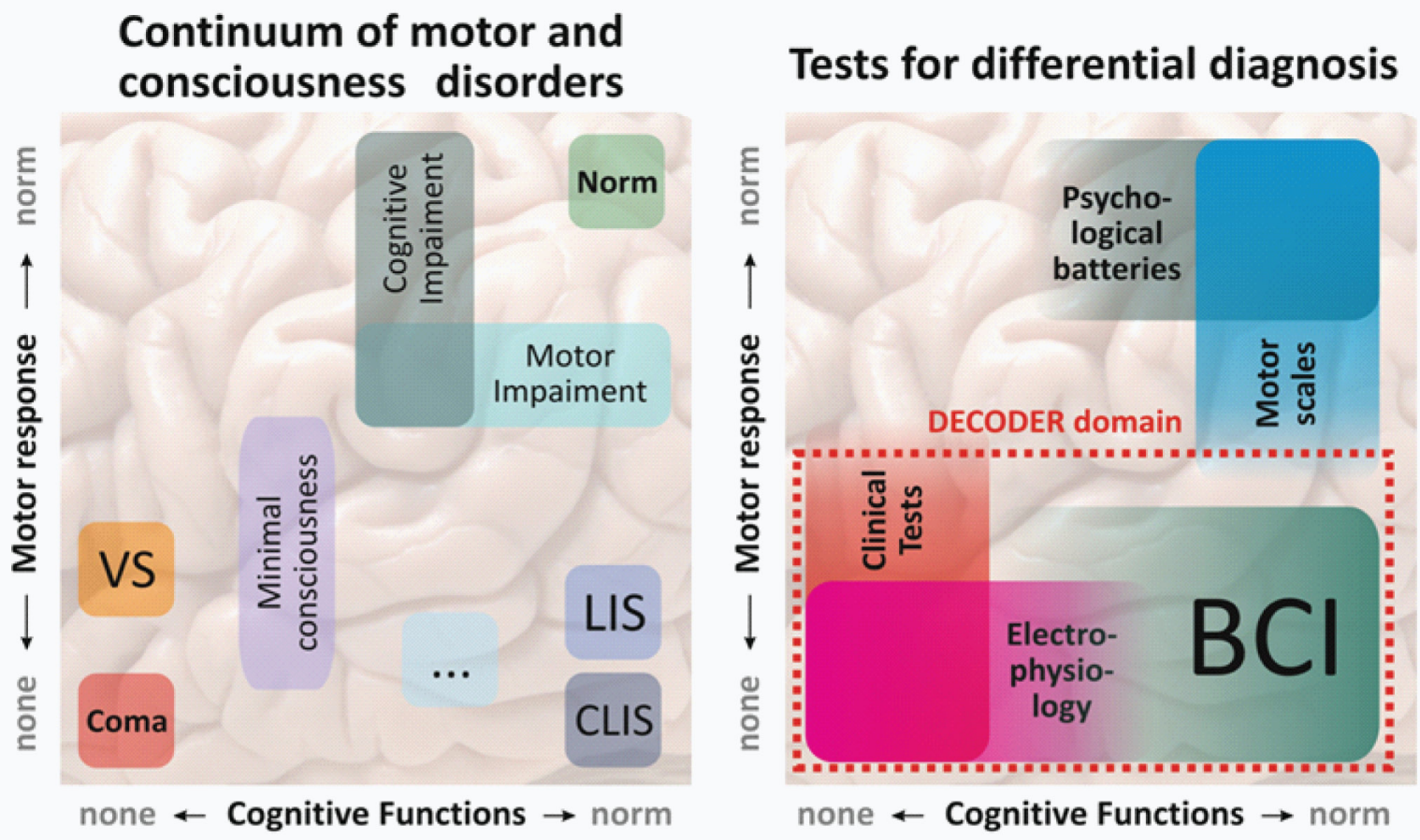

\section{Functional MRI to Assess Conscious Awareness and Communicate with Patients with DOC}

\section{Overview}

Although EEG-based systems are portable, and far less expensive than fMRI, fMRI has also been used successfully to develop a BCI system for patients with disorders of consciousness (DOC). Functional MRI measures the changes in blood oxygenation level of the brain, known as haemodynamics (Ogawa et al., 1993; Kwong et al., 1992). fMRI has several strengths for BCI applications, including its non-invasive nature, brain coverage and excellent spatial resolution (in the millimeter range).

Owen and colleagues (2006) employed an fMRI-based mental imagery paradigm to assess command-following in a patient who had been diagnosed behaviourally as in the VS, and had been unresponsive for five months. The patient was asked to imagine playing tennis (for 30 seconds) when she heard the word "tennis", and to relax (for 30 seconds) when she heard the word "relax." In a separate spatial imagery task, she was asked to imagine moving around the rooms of her home (for 30 seconds) when she heard the word "house", and to relax (for 30 seconds) when she heard the word "relax". The patient showed task-specific functional activation in the expected regions of the supplementary motor area (SMA) following the instruction to imagine playing tennis, and in the parahippocampal gyrus (PPA), the posterior parietal lobe (PPC), and the lateral premotor cortex (PMC) following the instruction to imagine moving from room to room in her house. Moreover, this activity was indistinguishable from that of healthy participants performing the same tasks (Boly et al., 2007; Owen et al., 2006). The patient's functional activation was statistically robust, reproducible, task-appropriate, and sustained over 
long time-intervals (30 seconds), allowing Owen and colleagues (2006) to conclude that she was responding to the commands by performing the imagery tasks in the absence of any overt action, and thus, that she was falsely diagnosed as being in the VS, albeit correct according to behavioural measures.

\section{Using fMRI to Achieve Binary Communication with DOC Patients}

Monti et al. (2010) extended this approach to demonstrate that fMRI could also be used to communicate with a behaviourally non-responsive patient who was assumed to be in the VS. One type of imagery (tennis or spatial navigation) was mapped to a "yes" response, and the other to a "no" response. A single neutral word "answer" was used to cue each response to a question. To decode the answers, each communication scan was compared to two 'localizer' scans, during which the patient was asked to simply imagine playing tennis or imagine moving around his house (see Owen et al., 2006). Following five autobiographical questions (e.g., "Is your father's name Thomas?"), the answers that were decoded from the brain activity matched the factually correct answers, which were unknown to the experimenters at the time (Figure

Figure 2. The top panel (1 - 2) shows the patient's brain activation when he is imagining playing tennis (left), and when he is imagining walking around his house (right). The patient's brain activity was indistinguishable from that of the healthy controls performing the same tasks. The bottom panel (3 - 4) shows the patient's brain activity when he was instructed to use these mental imagery tasks to convey either the word 'yes' or 'no', in response to being asked binary ('yes'/'no') questions. The patient's brain activation could unequivocally decode the answers to several autobiographical questions. The responses derived from the patient's brain activity were subsequently corroborated as factually correct by the patient's family.

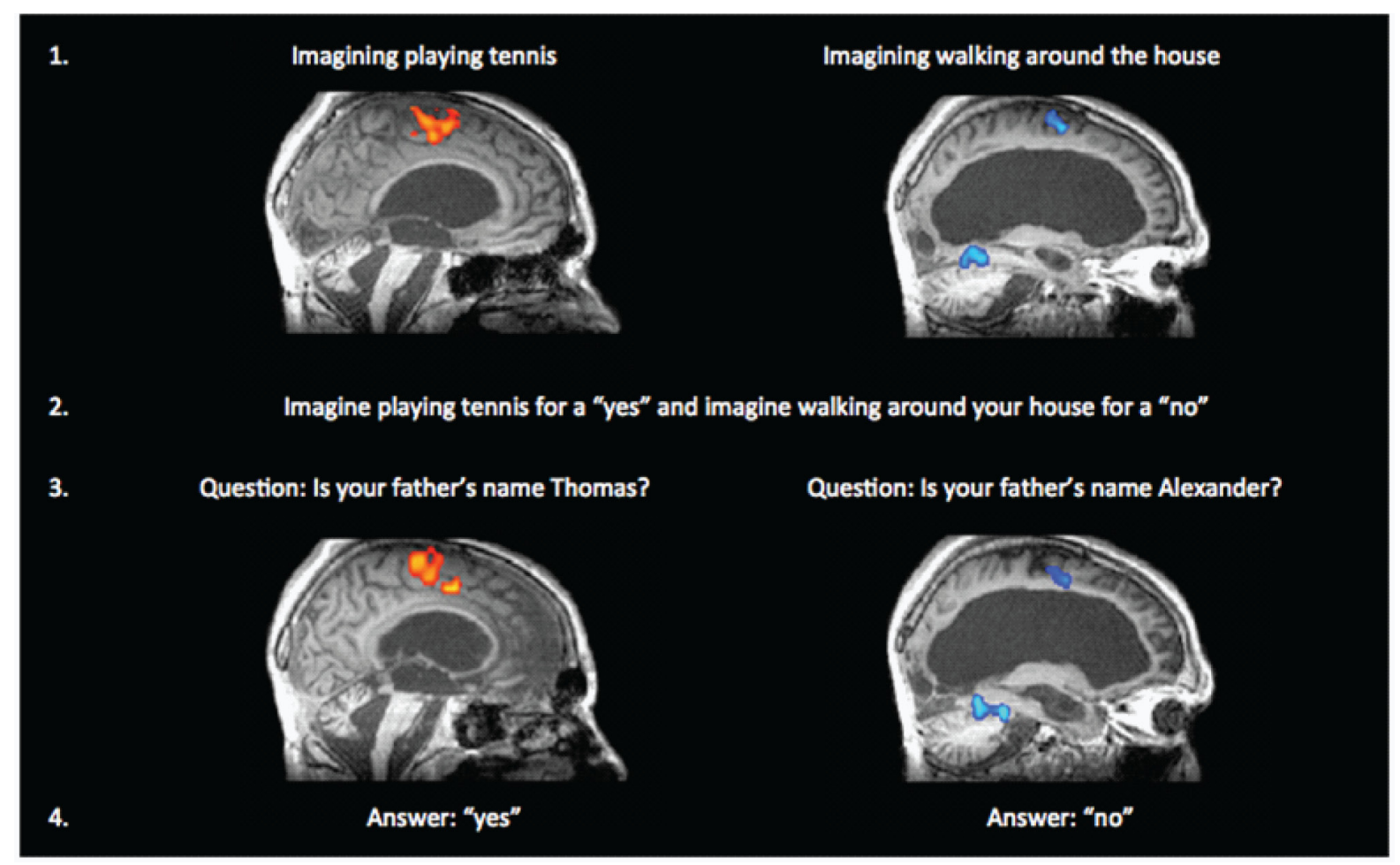


2). This study demonstrated that the presence of voluntary, reliable, and sustained brain activity in response to a command could be used as a proxy for physical behaviour, such as movement or speech, to facilitate communication with non-responsive participants (Owen and Coleman, 2008).

In the study described above, $54 \mathrm{VS}$ and MCS patients were tested and, of those, only five (four VS) showed significant changes in fMRI activation during the basic imagery tasks. One interpretation of this finding is that the diagnosis was accurate in the vast majority of cases, and the negative results reflect a genuine lack of awareness in those patients. Several other factors, however, may also explain these findings. First, this technique might lack sensitivity, and thus failed to show activation in patients who might have been engaged in the task (Bardin et al., 2011). Indeed, it is known that in brain-damaged patients, the coupling of haemodynamics and neuronal firing, which lies at the basis of the fMRI signal, may be very different from that in healthy volunteers (Rossini et al., 2004; Gsell et al., 2000). Alternatively, it is possible that, in some patients, deficits in language comprehension, decision-making, working memory or executive function may have hampered their efforts to express themselves through the imagery task, yielding brain activity too weak to be interpreted. Consistent with this possibility, a recent report found an MCS patient who showed no distinguishable activation in the mental imagery task, but, nonetheless, was able to voluntarily modulate his brain activity by allocating visual attention in response to verbal commands (Monti et al., 2013). Finally, in some patients, functional re-organization of the brain following the injury may have produced highly atypical patterns of fMRI activation, which may be impossible to interpret.

\section{Discussion and Future Directions}

Multi voxel pattern analysis (MVPA), an fMRI analysis technique that is highly sensitive to the information content in the neural signal, may provide a solution to some of these issues. Traditional univariate fMRI analyses average across activations in a brain region, and compare overall changes in signal strength between different types of conditions (Friston et al., 1995). MVPA, on the other hand, does not discard the information relating to the patterns of activity within that brain region. As such, it is capable of dissociating overlapping neural patterns to different stimuli or mental state (deCharms, 2007; 2008), which could not be disentangled with univariate methods (Haynes and Rees, 2006). By dissociating several mental states or responses elicited by a single command (Chadwick et al., 2010; Kay et al., 2008), MVPA also has the potential to expand communication from binary responses to multiple-choice answers. For example, while further in the future, MVPA could lead to tools that can help ask patients to express how much pain they feel on a sliding scale from 1-10 by imagining the appropriate number.

MVPA methods can also be applied in realtime (Caria et al., 2012; Sitaram et al., 2011; Lee et al., 2009; LaConte et al., 2007), and present exciting possibilities for communication without any perceptible delay between the question and the interpretation of the response. With these methods, however, classification accuracy is strongly dependent on the amount of available fMRI data. This may be a problem for VS patients, where the scanning time is often limited due to physical reasons. For example, patients may experience difficulty lying supine for long periods of time. Moreover, VS patients may become exhausted easily and are very likely to exhibit a very short attention span.

In summary, fMRI has great strengths for BCI applications, including its non-invasive nature, global brain coverage, and excellent spatial resolu- 
tion of specific brain structures, However, fMRI also comes with significant limitations, which restrict its widespread use in DOC patients. In particular, its high cost, lack of portability, and physical impositions on some patients (e.g. patients must not wear paramagnetic equipment, refrain from any minor movement, and be able to cope with the loud noise of the fMRI scanner), render this technology unlikely to provide the ultimate communication solution that DOC patients require in real life situations. Electroencephalography and fNIRS are not susceptible to such caveats, and provide exciting opportunities to promote and further develop the insight gained with fMRI.

\section{Conventional EEG Methods}

Unlike fMRI, EEG is easy to set up, portable, widely available and inexpensive. EEG is one of the most important neurophysiological methods to assess cognitive functions (e.g., attention, language, etc.) in humans. These features make it practical for bedside testing. EEG also can measure brain activity with excellent temporal resolution. One common method of analyzing EEG data is to extract event-related potentials (ERPs) from the recorded EEG. An ERP is a specific brain pattern that is time-locked to a specific event, such as a flash, tone, or movement (Luck, 2005). In general, ERPs reflect the synchronous activity of postsynaptic potentials produced by the parallel firing of cortical pyramidal neurons during information processing (Peterson et al., 1995). In healthy participants, those reactions are elicited reliably and are well-studied. Based on this, they can also serve as important indicators of cognitive function in severely disabled patients with DOC (Kotchoubey et al., 2005, D’Arcy et al., 2011).

EEG can indeed be useful for assessing cognitive state and for communication. One recent study found that 3 of 16 patients (19\%), who were classified as VS and totally unresponsive behaviourally, could consistently produce specific EEG patterns to two different commands (Cruse et al., 2011). These results show that EEG methods could help identify VS patients who might be able to communicate with an EEG-based BCI. However, developing practical and reliable tools in field settings entails many challenges such as patients' low attention span, demanding set-up, and electrically noisy environments.

\section{The Hierarchical Approach and Relevant ERP Components}

The hierarchical approach is regarded as the most effective way to examine cognitive functioning in DOC patients (Kotchoubey et al., 2005; Kübler and Kotchoubey, 2007; Owen et al., 2005). Figure 3 depicts the interplay of the three suggested levels of the hierarchical approach investigating different cognitive functions underlying consciousness. Different EEG paradigms, each of which elicits specific ERP components, are associated with each of the levels.

The first level includes the assessment of basic cognitive functions. These functions may be completely unconscious, but this level is critical before moving to the next level. Within the first level, paradigms test the patient's sensory and automatic cognitive information processing capacities, which are reflected in the ERP components P1/ N1 (sensory input) and the mismatch negativity (MMN). Typically, the MMN is elicited by a rare stimulus, called deviant, that differs from the frequently-occurring stimuli, called standards, in one or more physical parameters such as duration, intensity, frequency or location (oddball paradigm; Näätänen et al., 2004; Schröger and Wolff, 1996; Jacobsen and Schröger, 2003). The MMN is in- 
Figure 3. Hierarchical approach with three different levels of cognitive processing and their respective EEG/ERP indicators
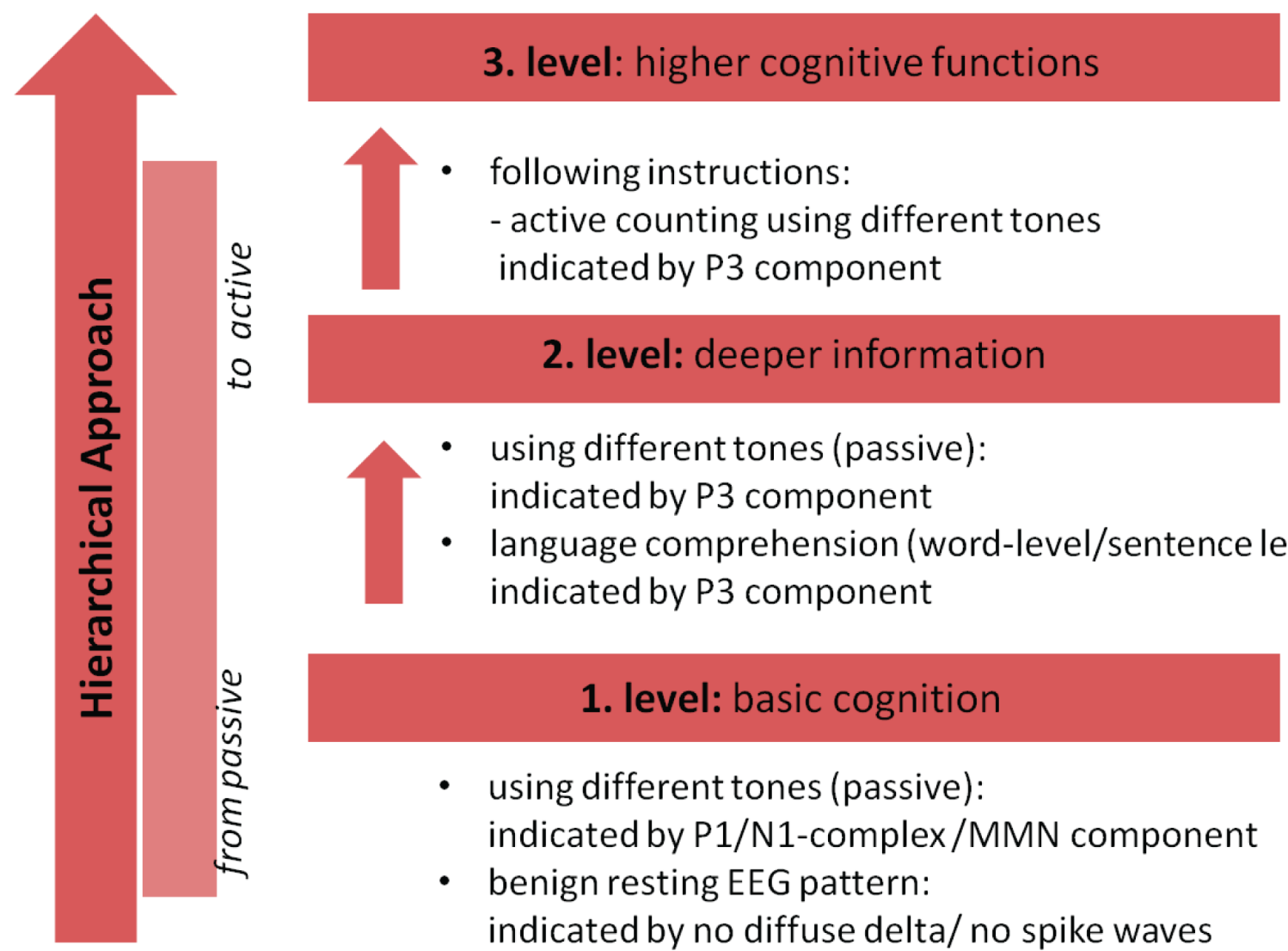

- using different tones (passive): indicated by P3 component

- language comprehension (word-level/sentence level): indicated by P3 component

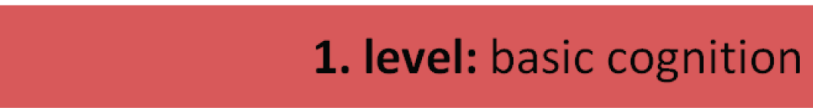

- $\quad$ using different tones (passive): indicated by $\mathrm{P} 1 / \mathrm{N} 1$-complex/MMN component

- benign resting EEG pattern: indicated by no diffuse delta/ no spike waves

terpreted as an index of a mismatch between the actual stimulus and the memory trace of physical or abstract environmental regularities. Thus, it appears in response to a violation of simple rules or expectations (for review, see Näätänen et al., 2007). The important characteristic of this violation detection is the high level of automaticity. MMN paradigms can be used successfully in nonand low-responsive patients (Kane et al., 2000; Guérit, 2005) because MMNs do not depend on the actual cognitive state of a patient (e.g., fatigue, monotony) and the daily fluctuations of arousal (Kotchoubey et al., 2003). Therefore, the MMN is a good predictor toward a positive or negative outcome of comatose patients (e.g., Fischer et al., 1999, see also meta-analysis by Daltrozzo et al.,
2007) as well as for the improvement of VS patients (Kotchoubey et al. 2005; Wijnen et al., 2007).

The second level can reveal deeper information processing that indicates, though does not prove, conscious intentional states. One way to test this level is to assess language related processes, reflected in the N400 ERP component (e.g., Kutas \& Hillyard, 1980). The N400 is strongly correlated with semantic manipulation (Hagoort, 2005; Kutas \& Federmeier, 2011; Sitnikova et al., 2002) and is distributed over centro-parietal areas. The semantic manipulation can be achieved on the word level by presenting word pairs which are related (mountain-valley) or unrelated to each other (place-bravery; Hagoort et al. 1996) and on the sentence level by presenting sentences with 
correct word endings ("The eel is aquatic.") and incorrect word endings ("The eel is a bird."; Connolly et al. 1992). Thus, a more pronounced N400 component for unrelated/incorrect conditions compared to the related/correct conditions indicates the ability of low- and non-responsive patients to process complex language information (Kotchoubey et al., 2002). Recently, research also showed that correctly and falsely negated sentences (birds cannot speak vs. birds cannot fly) elicit an N400 of a different amplitude (Herbert and Kübler, 2011). As negation requires cognitive as opposed to automatic processing, this paradigm may be regarded as transitory between the second and the final level.

Finally, the third level includes higher cognitive functions (e.g., selective attention, instruction understanding). Only this level can reveal strong indication of conscious awareness. One ERP component important for this and the previously described second level is the $\mathrm{P} 3$ (or P3b). The P3, like the MMN, is often investigated in the context of the oddball paradigm (Duncan-Johnson \& Donchin, 1977). The $\mathrm{P} 3$ can be elicited without an active instruction, especially to stimuli of particular significance, like a subject's own name (Fischler et al., 1987, Kotchoubey et al., 2005). However, the P3 increases substantially when subjects are instructed, for example, to count a rare stimulus in a sequence containing frequent and rare stimuli (Lang et al., 1997). Thus, passive $\mathrm{P} 3$ paradigms indicate the level two in the hierarchical approach, whereas active $\mathrm{P} 3$ paradigms reflect the third level. These paradigms may serve as a battery for the assessment of the level of consciousness (BAC). The following section presents recent research that shows the usage of this hierarchical approach.

\section{CHALLENGES AND ISSUES WITH EEG-BASED METHODS}

The previous section demonstrated that, despite substantial research and development, conventional tools for assessing DOC are inadequate. This section discusses dominant issues, methods and paradigms in EEG-based assessment of consciousness. We discuss some limitations of current methods based on interviews and empirical work. Next, we address problems with conventional analysis methods with single subjects and single trials.

\section{Requirements for an EEG- Based Diagnostic Battery for Patients with DOC}

Above in this chapter, the major problem of misdiagnosis in DOC patients was largely discussed from the point of view of neuroscience and the results of neuroimaging research. However, the perspective of practicing clinicians is also of importance. We performed a study to assess clinicians' views regarding current diagnostic procedures and possible improvements. In the past years, studies have repeatedly indicated a high proportion of misdiagnoses in patients in minimal conscious (MCS) and vegetative state (VS) (Schnakers et al., 2009). Thus, diagnosis in such low or non-responsive states needs to be improved, and ongoing research aims at developing diagnostic means based on imaging and electrophysiological techniques. Event-related potentials (ERP) have been shown to be a suitable tool to complement clinical assessment and to detect residual cognitive functions (Kotchoubey at al., 2002). However, such tools have to meet the requirements of physicians in the field and must be developed in close collaboration with 
these practitioners, who are the target user group for such an EEG or imaging based diagnostic battery. Following a user-centered approach in which a product is developed in an iterative process between users and developers (e.g., Zickler et al., 2011; Holz et al., 2012; Kübler et al., 2013), nine semi-structured interviews were conducted with representatives from acute care clinics (ACC, 4) and neurological rehabilitation centers (NRC, 5) across Germany.

Interviewees were five medical directors, two chief physicians and two senior physicians. They were told about the development of a new EEG-based diagnostic battery based on auditory ERPs. The interviews covered three main topics: current diagnostic procedures, weaknesses of the current process and expectations concerning a new diagnostic battery. All interviews were recorded and transcribed. Answers to each question were grouped into clusters and counted (Erlbeck \& Kübler, 2013).

In general, results supported the hypotheses that clinicians agree that current procedures need improvement. For clarity, answers of ACCs and NRCs will be presented together unless otherwise noted.

\section{Current Procedures}

A diagnosis in ACCs is made rather quickly within few hours. NRCs acknowledge the diagnosis provided by the referring ACC but review it on admission of the patient. A diagnosis is regularly checked on an hourly (ACCs), daily or weekly (NRCs) basis depending on the status and medical history of the patient. When making a diagnosis, physicians primarily rely on the clinical assessment, meaning the observation of reactions to auditory, sensory and visual stimuli. All institutions apply EEG, especially in comatose, VS and MCS patients. One institution (NRC) also utilizes measurements of evoked potentials. In some cases, CT $(n=6)$ or fMRI $(n=4)$ are also used. The Glasgow Coma Scale is administered in six, the Barthel Index in five and the Coma Recovery Scale revised (CRS-r) in two institutions. Seven institutions consider their diagnosis to be of primary importance for treatment decisions and future therapeutic processes. Two institutions (NRC) consider the diagnosis to be important but put a greater focus on prognosis and treatment.

\section{Weaknesses in the Current Procedures}

Results in the current diagnostic process partially depend on experience and observational skills of the responsible physician $(n=5)$. Therefore, there is interest in a stronger focus on different aspects of diagnostics in the education of becoming a neurologist $(n=3)$. Another critical issue is the lack of methods to estimate the further development of patients in terms of regaining consciousness or rehabilitative progress $(n=3)$. Furthermore, a lack of sufficient resources to apply imaging techniques was mentioned $(n=3)$. Finally, the consideration for psychological matters and cognitive performance (as the entity that makes us humans) is considered insufficient in neurological diagnostics $(n=2)$.

\section{Requirements and Expectations}

A general interest in applying a new, more reliable diagnostic tool was evident in many institutions (one ACC, five NRCs). Three representatives from ACCs could not imagine using it in their institutions but consider it interesting, particularly for therapeutic institutions such as NRCs. All interviewees named reliability and validity as mandatory. Additionally, the tool must not be affected by disturbances typical in a medical environment $(n=6)$ and must be practical in terms of time, personnel, financing and the setup $(n=7)$. Respondents also mentioned that the amount of resources available to invest will largely depend on the benefit of the resulting output $(n=4)$. Taking into account the weaknesses of the current 
diagnostics, the most relevant expectations are the prognostic value of the results $(n=7)$ and a support in therapeutic decision-making $(n=5)$. Thus, the output is expected to be accurate in terms of a selective differentiation between various diagnoses and prognoses.

Overall, a correct diagnosis is vital for minimally or non-responsive patients, not only because prospects for MCS patients are more favorable than for VS patients but also to ensure that consciousness in a non-responsive patient is detected (Healy, 2010). The results reveal that physicians are aware of weaknesses in the current diagnosis process and want a new diagnostic EEG-based tool. It must be highly valid, work reliably, allow for prognostic statements and not add to the burden of limited financial and personnel resources. The necessity of such an EEG-based tool to improve reliability of the diagnosis is widely acknowledged as current clinical assessment is influenced by individual skills of the physician.

To conclude, a practical, reliable and valid EEG-based diagnostic tool would be highly welcome in clinical and rehabilitative routines. Thus, the ERP-based paradigms to delineate the level of consciousness and cognitive function in otherwise non-responsive patients need to be validated such that only the most reliable and informative are applied when time is limited. The decision of which paradigm works best for which level of consciousness or cognition requires normative studies with representative healthy samples. The prognostic value of such paradigms can only be determined in longitudinal studies. A preliminary study with the BAC in a small sample of patients is described in the next section.

\section{Tests on Patients with DOC and Locked-In Syndrome}

The ERP paradigms proposed above were tested on a group of DOC and LIS patients. One reason to study LIS patients is to establish patterns of evoked potentials within BCI paradigms in brain injured patients who do not exhibit disorders of consciousness but are behaviourally quite similar to non-responsive patients.

\section{Methods}

Here we describe and compare the results of 6 DOC patients in acute and chronic stages, recruited in the Post-Coma Unit of the Neuro-Rehabilitation Hospital "Fondazione Santa Lucia" in Rome, and of 9 chronic LIS patients ( $>1$ year in LIS, 6 female, 3 male, mean age $40 \pm 9$ years) from the French Association for Locked-in syndrome (ALIS) tested with MMN, and the passive and active $\mathrm{P} 3$ of the BAC developed within the DECODER project. Diagnosis of VS $(n=3)$ and MCS ( $n=3)$ was based on the CRS-r. EEG was recorded with an active 32-electrode cap with two biosignal amplifiers g.USBamp (g.tec medical engineering $\mathrm{GmbH}$, Austria) and recordings were processed and analysed with the NPXlab2012 software (www.brainterface.com). Data were preprocessed with Independent Component Analysis (ICA). Independent components corresponding to ocular artifacts were removed and other artifacts were removed by means of a threshold criterion (i.e. signal segments exceeding $70 \mu \mathrm{V}$ in absolute value). Components of interest were analyzed by visual inspection and by running a t-test. Differences were considered statistically significant for $\mathrm{p}<0.05$ if they appeared simultaneously at least on two electrodes and they lasted a minimum of 50 consecutive milliseconds.

\section{Results}

With regards to DOC patients, the Mismatch Negativity (MMN) was observed in three of six patients and the $\mathrm{P} 300$ was present in all but one patient in both the active and the passive conditions (Table 1). In one LIS patient, tests were stopped due to fatigue. Data from two patients (3 and 7) were discarded due to excessive artefacts. In the six remaining LIS patients, four presented 
Table 1. Results from the 6 DOC and the 6 LIS patients analyzed. $(+)=$ presence of ERP component; $(-)$ = absence of clearly recognizable ERP components.

\begin{tabular}{|l|c|c|c|}
\hline & MMN & P300 Passive & P300 Active \\
\hline MCS 1 & + & + & + \\
\hline VS 2 & + & + & - \\
\hline MCS 3 & - & + & + \\
\hline MCS 4 & - & + & - \\
\hline VS 5 & - & - & + \\
\hline VS 6 & + & - & + \\
\hline LIS 1 & - & - & + \\
\hline LIS 2 & - & + & + \\
\hline LIS 4 & - & + & - \\
\hline LIS 6 & - & + & - \\
\hline LIS 8 & - & - & + \\
\hline LIS 9 & - & & + \\
\hline
\end{tabular}

an active $\mathrm{P} 3$; three a passive $\mathrm{P} 3$; and none had an MMN (Table 1).

\section{Discussion}

The auditory P300 and the MMN can offer a valuable supplement to early routine clinical examination and prediction of neurological outcome of post-comatose patients. From the data collected in this small patient sample, it seems that the presence of the considered components is not related to the severity of the disorder of consciousness, but rather to the different states of the brain, in terms of site and extent of the lesions. The MMN component points to a sufficiently spared preattentive sensory memory system (Näätänen et al., 2007), while the presence of the $P 3$ suggests that working memory updating (Coles et al., 1988) is functioning in most of these patients.

Results in LIS patients revealed a response to the higher cognitive function paradigm (the active P300 task) even in the absence of the MMN and P300 in the passive condition. Regarding the MMN, a reduction of this ERP component has been described in patients with cognitive deficits (Duncan et al., 2009). In LIS patients, a study has shown a moderate and selective cognitive impairment that is not related to the location of the lesion (Rousseaux et al., 2009). However, this does not explain the absence of an MMN in LIS patients. In this patient sample, the absence of lower level components (see Figure 3) and even the passive P300 did not indicate the absence of higher level components (active P300). This has important implications for the diagnosis of altered states of consciousness, confirming previous work (Kotchoubey et al., 2005) that this hierarchical approach is flawed.

\section{The Hierarchical Approach in Clinical Assessment}

Another concern with the hierarchical approach involves its use within the daily clinical routine. For example, is it appropriate to stop assessing patients after one or two paradigms (corresponding to the first and second level) that do not yield promising ERPfindings? Figure 4, like the preced- 
ing section, indicates that stopping assessment so early is not justified by the data. This minimally consciousness state (MCS) patient (male, 35 years, coma onset 253/254 days) was examined twice using the same hierarchically ordered set of paradigms. The results revealed no correlation between both semantic paradigms (a positive ERP outcome in the word pair paradigm was not a prerequisite for the sentence understanding paradigm) and no correlation between the passive and active frequency oddball paradigms (a positive ERP outcome in the passive condition was not a prerequisite for the active condition). Thus, no informative conclusion about the present state of the patient could have been drawn if he were tested only with a single paradigm. However, a prerequisite of such an assessment is the patient's ability to generally perceive sensory stimuli. Thus, further EEG analysis appears meaningless only if a frequent (standard) tone does not elicit even a primary cortical ERP complex P1-N1-P2. Otherwise, all passive paradigms and at least one active oddball or one more active paradigm (e.g., dichotic listening, Yvert et al., 1998; a semantic oddball, Kotchoubey \& Lang, 2001; or negated sentences, Herbert \& Kübler, 2011) should be conducted in each patient before a final conclusion about the level of consciousness can be drawn.

In addition, this example shows that several measurements within one patient should be performed to increase the reliability of the assessment. Repeated measurements help to minimize error due to the well-known phenomenon of fluctuating arousal and attention in these patients. Further-

Figure 4. ERP responses of a VS patient. The black arrows point to significant components in which the two stimulus classes differ within an auditory paradigm - specifically, the P3, N400 and MMN.

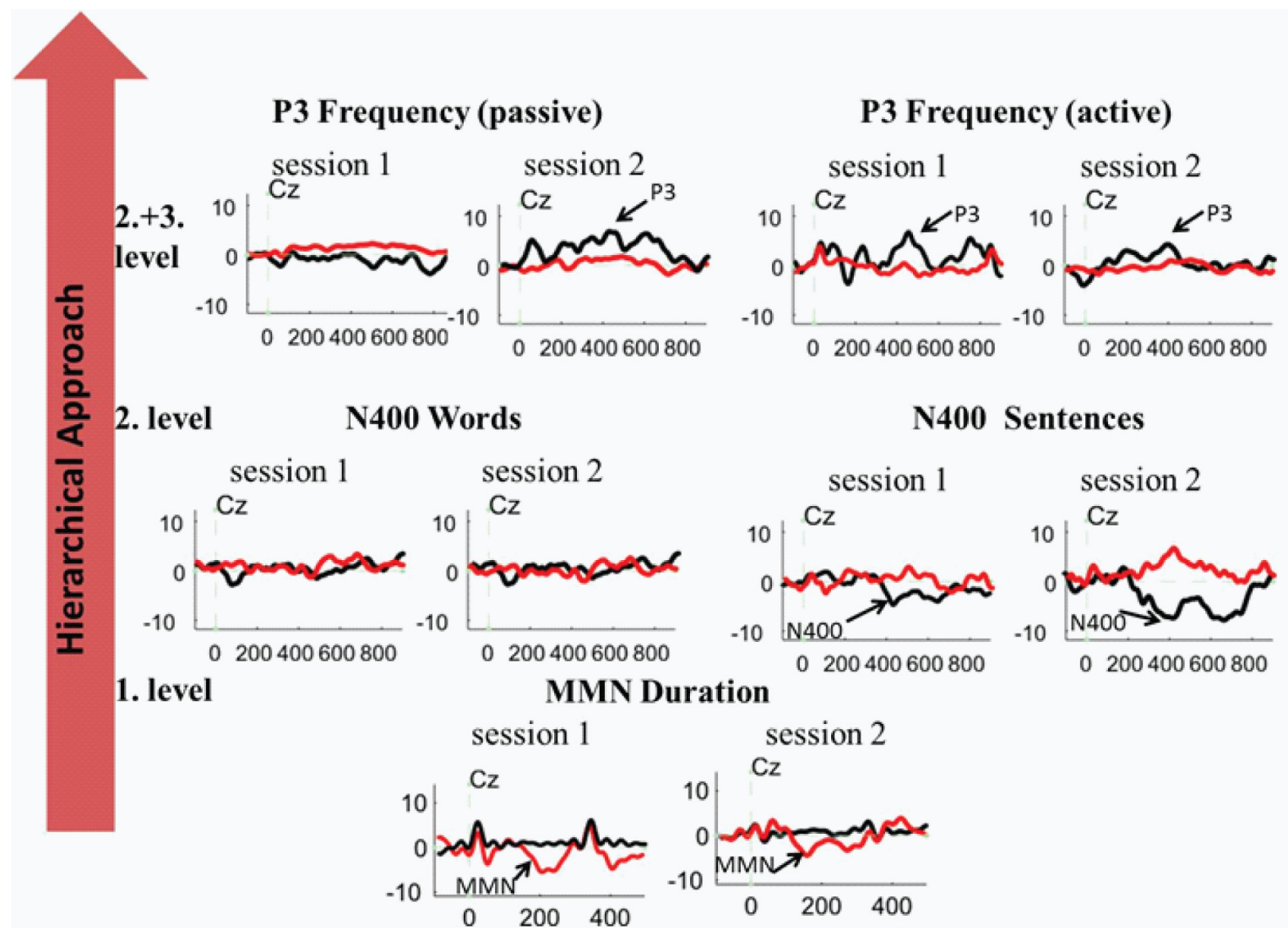


more, the development of appropriate paradigms for low- or non-responsive patients requires a difficult tradeoff between the reliability of the signal and the capacities of the patient. The longer the experiment is, the better the signal-to-noise ratio and, thus, the higher the data quality. However, the attention span of the patients is very limited and they might be overchallenged by long sessions. This balancing act is difficult and small estimation errors could yield misleading, but consequential results. Moreover, differences in ERP responses of patients need to be judged on the single subject level, whereas the ERP responses elicited by the paradigms described above are typically analyzed on the group level.

\section{Analyses on a Single Subject Level: Concerns and Solutions}

Traditionally, the presence and absence of ERP components are analyzed with respect to measures such as latency, polarity, topography and sensitivity to experimental manipulations (Picton et al., 2000). However, nearly all ERP research involves healthy subjects. These measures may be too weak to detect whether the experimental manipulation had an adequate effect on the mean amplitudes of P3 components (shown in Figure 4), because ERPs in brain-injured patients may occur with prolonged latencies, and, depending on morphological changes in brain and skull structure, with altered topography and polarities (Duffy, Burchfiel, \& Lombroso, 1979 Kotchoubey, 2005; Duncan et al., 2009). Thus, analysts might erroneously interpret any differences between amplitude curves (elicited by two different experimental conditions like standard and deviant) as indicators of different brain processes. Conventional methods such as t-tests between two conditions at each sample and electrode positions may lead to unacceptable alpha inflation. Hence, we describe three correction procedures for the single-subject analysis of EEG data.
1. False discovery rate (FDR) is a term for various procedures designed to control the expected number of false positive findings (Groppe et al., 2011). Compared to classical procedures, e.g. Bonferroni's procedure, which control the family wise error and can be very conservative with large numbers of comparisons, FDR procedures provide adaptive error control, depending on the number of significant results. However, this approach may be problematic when trying to identify small differences in the absence of other, large differences, which might happen when analyzing data in a priori defined time-windows. Thus, effects declared as significant using FDR procedures might be difficult to replicate when using a priori defined time windows (Groppe et al., 2011).

2. Another procedure was originally proposed by Guthrie \& Buchwald (1991). This method takes into account that consecutive EEG samples - and any derived measures, such as t-values - are not independent, but show a high autocorrelation. By using an empirically derived estimation of this autocorrelation, it is possible to define a minimum length for the duration of series of values exceeding a predefined cutoff. In contrast to FDR, the effectiveness of Guthrie-Buchwald's procedure does not depend on the magnitude of observed effects, but on their duration. Thus, this method is most effective to detect long drawn out differences between two ERP curves, like the P3. Conversely, it is not suited for the detection of short differences, as the probability of short series might be judged to be too high, assuming a given autocorrelation. Further, Blair \& Karniski (1993) have highlighted the problem of, among others, accurately estimating the autocorrelation of EEG data.

3. The Studentized Continuous Wavelet Transform, originally proposed by Bostanov \& Kotchoubey (2006), analyzes EEG data 
in the two-dimensional time-frequency domain. First, the continuous wavelet transform (Mallat, 2007) is calculated for each segment of EEG data. Second, a statistical map is created by calculating the $t$-values associated with the wavelet coefficients at each point in the time-frequency plane between two experimental conditions. Third, local extremes are detected in this statistical map. Finally, $t$-max randomization tests are used to determine which local extremes represent true vs. false differences in activation.

Each method has strengths and weaknesses. Thus, it is important to realize that effective diagnosis and analysis depends on both sensitivity and specificity. Any imbalance, such as high sensitivity but low specificity (when using multiple unadjusted comparisons) or low sensitivity but high specificity (when using FDR (Bonferroni) corrections) is likely to impede progress of the assessment (Real et al., 2013).

\section{ERP Analyses of Single Trials: Concerns and Solutions}

There is a growing interest in decoding ERP components at the level of single trials. This could be useful in many ways, such as identifying mental states or user's intentions so BCIs can be applied for medical purposes. However, this issue remains very challenging due to the extreme trial-to-trial variability. One way to deal with the problem of single-trial detection of relevant components of the EEG signals is the use of detection algorithms, also called classifiers. Classifiers are mathematical tools designed to accurately recognize a relevant feature from single trial data to ultimately estimate if the brain can distinguish between two or more external stimuli (e.g. deviant versus standard). Once the accuracy of such a discrimination process is computed, its statistical significance with respect to the chance level can be evaluated by means of statistical tests (e.g. chi-square test). The significance of the accuracy strictly depends on the number of classified trials and is measured by means of a $\mathrm{p}$-value. This procedure can provide an index about the reliability of the discriminated responses, and thus it could support clinicians in DOC patients' evaluation. Relevantly, such methodology is independent from the used protocol.

To better illustrate the potential advantage of such a procedure for medical applications of BCIs, like the detection of mental processing in absence of behavioral signs, we present results of its application on EEG data sets obtained from two patients diagnosed as VS. They were administered a classical acoustic oddball P300-based paradigm ( $2^{\text {nd }}$ level of the hierarchical approach), during which they were asked to mentally count the deviant tones (targets, $\mathrm{T}=60$ ) against the standard (no-targets, NT=420). Significance threshold was set at $5 \%$. In one patient, the classifications (stepwise linear discriminant analysis -SWLDA) across 109 trials were random $(\mathrm{p}>0.05)$ for both target (49.06\%) and no-target $(60.71 \%)$ classes. In the second VS patient, the accuracy was $65.38 \%$ and $78.33 \%$ for target and no-target classes, respectively. Both classes were significantly classified $\left(\mathrm{p}_{\text {target }}=0.027, \mathrm{p}_{\text {no-target }}=0\right)$.

It is mandatory to stress that the absence of a positive result, as in the firstcase, could be ascribed to many factors independent from the diagnosis of VS, thus preventing any definitive conclusions with regards to the clinical diagnosis. False negative inducing factors may be: vigilance status at the time of the recording (patient was sleeping); cognitive function masked by the DOC that affects the auditory task accomplishment; high signal to noise ratio; or the classifier was not effective enough to extract relevant features. One recommended solution is to conduct multiple recordings, as described above, at different times and/or to test different translation algorithms to identity the best appropriated for the relevant features. On the other hand, a possible occurrence of false 
positives (statement on the presence/intactness of patient's cognitive processing ability) has to be considered. In this case, one source of errors might be the capture of brain response components not directly related to a voluntary shifting of attention between the two classes (target and no-target) that interfere with the algorithm classification output. A careful stimulation protocol design is the first step to prevent such an error source.

Overall, this section provides further support for the view that conventional DOC assessment procedures are inadequate, and that EEG-based measures can provide an effective, standard and easy methodology to detect residual cognitive activity in DOC patients. However, progress is hampered by procedural errors, which we discussed along with some solutions. The next section introduces the mandatory step for DOC patients provided consciousness was detected, i.e. a means of communication.

\section{$\mathrm{BCl}$ : The Challenge and a Possible Solution}

There are many challenges in developing a system that can detect consciousness, particularly with individual patients and single trials. However, if a non-responsive patient is consciously aware, then a new challenge arises: how can we establish communication in such a patient? A single-switch BCI (ssBCI) may be able to detect one specific brain pattern of a patient and use it to control different kinds of applications. Since each patient will react differently, the DECODER project explores several brain signals.

When designing a BCI for non-responsive patients, the specific cognitive characteristics of these patients have to be considered. Therefore, the BCIneeds to be very simple and robust, meaning it should function even if only a single brain pattern can be reliably detected in that patient. Since individual patients will react differently, mental imagery (Horki et al., 2012; Müller-Putz et al., 2013), P300 (Müller-Putz et al., 2012;
Pokorny et al., 2013), and steady-state evoked potentials (Pokorny et al., 2011; Lesenfants et al., 2013) were explored. The P300 paradigm was implemented with auditory and vibrotactile stimulation. As examples, the methods for the auditory BCI and for the motor imagery based $\mathrm{BCI}$ will be described here.

\section{Methods and Signal Processing}

In the auditory P300 paradigm, two different tone streams composed of short beep tones with infrequently appearing deviant tones at random positions were used as stimuli (Müller-Putz et al., 2012; Pokorny et al., 2013). The streams were presented simultaneously with asynchronous onsets. By intentionally shifting attention from one stream to the other, the P300 response elicited by the deviant tones in the attended stream should be modulated. This paradigm was evaluated in ten healthy subjects and applied to twelve MCS patients (four female, eight male), aged between 14 and 66 years, at four different locations. EEG measurements were conducted in Graz (Albert Schweitzer Clinic), Würzburg (Intensive Care Hospital Schwaig), Rome (Fondazione Santa Lucia) and Liège (CHU University Hospital). This study was approved by the local Ethics Committees at all participating institutions and informed consent was obtained from the patients' legal representatives. A SWLDA classifier together with $10 \times 10$ cross-validation was used to infer which tone stream was attended. Moreover, all data segments from one participant were averaged according to stimulus type and target stream, and significant differences ( $\alpha=5 \%$, length $\mathrm{L} \geq 30$ ms) between (i) standard and deviant tones and (ii) target and non-target deviants were estimated by bootstrapping using 1000 bootstrap samples.

In the mental imagery paradigm, we explored whether complex mental imagery and attempted feet movement could be reliably detected in DOC patients (Horki et al., 2012; Müller-Putz et al., 2013). Four male patients in MCS aged 
$21-65$ years participated in this study at Albert Schweitzer Clinic in Graz. Informed consent was obtained from the patients' legal representatives. This study was approved by the Ethics Committee of the Medical University of Graz. The participants were instructed to perform the following tasks: sport - imagine performing one sport of your choice in the first person perspective; navigation - imagine navigating through your house, and looking around each room; feet - attempt (repeatedly) feet dorsiflexion. Fisher's linear discriminant analysis (LDA) was used as a classifier based on logarithmic band power features calculated for multiple frequency bands ( $\alpha$ : 8-13 Hz; $\beta \mathrm{L}: 13-19 \mathrm{~Hz} ; \beta \mathrm{M}: 19-25 \mathrm{~Hz} ; \beta \mathrm{U}$ : 25-30 Hz). A nested blockwise cross-validation (10x10 inner fold; leave-one-block-out outer fold) was applied to estimate the classification accuracy of each task versus reference.

\section{Results}

In the P300 paradigm (Müller-Putz et al., 2012; Pokorny et al., 2013), results for healthy subjects were promising, and most classification results were clearly better than random. However, single trial classification results were above chance level in only one of the twelve MCS patients. In four of the patients, the presence of a P300 could at least be detected on a single trial basis when presenting only one of the tone streams. However, none of the results were sufficient for communication purposes. Nevertheless, signs of consciousness were detected in most patients, not on a single trial basis, but after averaging of corresponding data segments and computing significant differences (Figure 5A).

In the mental imagery paradigm (Horki et al., 2012; Müller-Putz et al., 2013), one patient showed patterns of activation over sensorimotor are as during at least one task involving either simple (i.e. feet dorsiflexion) or complex (i.e. sport) motor imagery (Figure 5B) in each of the two sessions. Two other patients showed patterns of activation over central sensorimotor area for complex motor imagery task in the first session. In one of the four patients, no significant results were found.

\section{Evaluating Protocol Performance with a BCl-Based Approach}

A BCI-based methodology was implemented to evaluate the used protocols. This methodology is

Figure 5. Examples of brain patterns found in minimally conscious patients. The two panels show significant differences that reflect mental awareness. (A) Significant P300 at channel Fz using the auditory P300 paradigm. (B) Significant ERD differences at channel C2 (orthogonal Laplacian derivation) in the alpha band using the mental imagery paradigm.
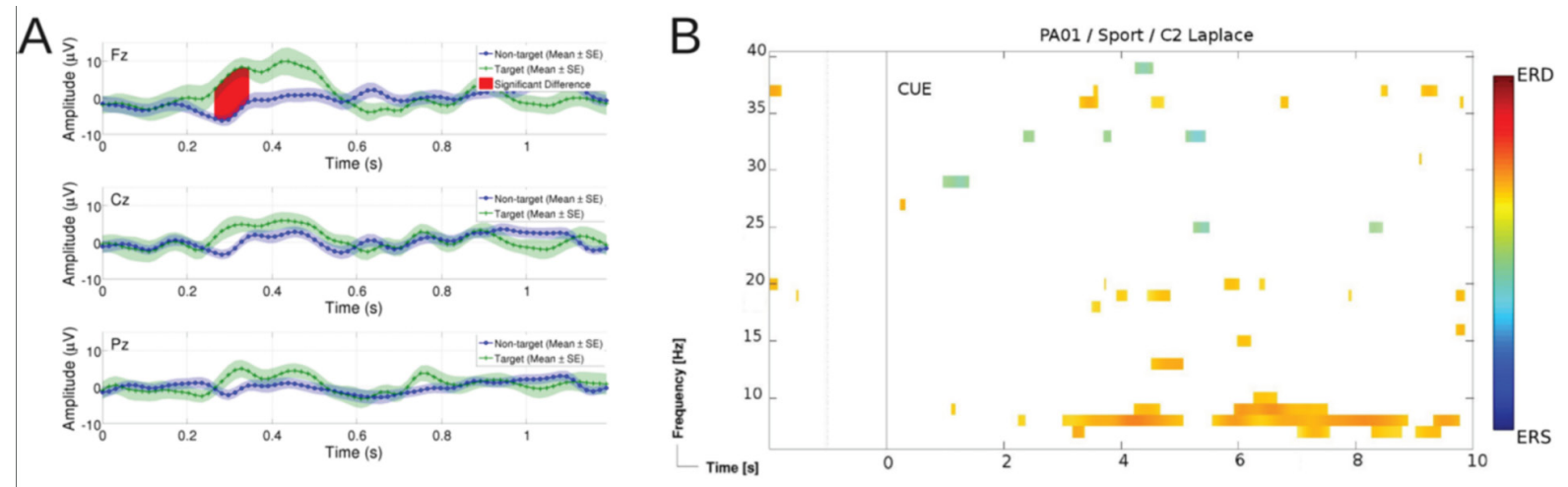
standardized, as it is independent from the protocol itself, the extracted features, and the used classifier. It is based on the evaluation of classification performances stored into a confusion matrix (CM) (Bianchi et al., 2007), and also on the assessment of the statistical reliability of classification accuracy. Briefly, once the classification accuracy is computed, by considering the number of trials in each classification class (sport, navigation, feet, target, non target, etc.), it is possible to assess how much such accuracies differ from what a random classifier would return (Billinger et al., 2013; Yuan et al., 2013). This information is complementary to the data obtained from the study of brain patterns, as it allows researchers to understand the significance and reliability of a detected brain response. Furthermore, it could help to identify the best task to setup the ssBCI based on the residual skills of each patient.

\section{Discussion}

In the auditory $\mathrm{P} 300$ paradigm, single-trial classification results were not sufficient for communication purposes (Müller-Putz et al., 2012; Pokorny et al., 2013). Nevertheless, since significant deflections were found on an average level, this paradigm might still be useful to support clinical assessment of patients, and to provide them with a means of communication. In principle, since time is not a crucial factor for unresponsive patients, communication could be established by simply averaging data over many trials and detecting significant differences due to attention. Toimprove the paradigm, different stimuli (e.g., words instead of tones) that may be easier to distinguish or elicit a stronger P300 response might be beneficial in the future (Ricci et al., 2013).

Using mental imagery, on the other hand, seems to be a more promising approach for some patients to communicate their intent (Horki et al., 2012; Müller-Putz et al., 2013). Classification accuracies above chance were estimated for the feet and/or the sport mental imagery, but not for the navigation task. This is in line with previous findings indicating that, among other tasks, motor imagery rather than spatial navigation most frequently results in better classification performance (Friedrich et al., 2012).

\section{Conclusion and Future Directions}

Another method that may work well for detecting command following and testing communication in non-responsive patients is based on modulation of steady-state visually evoked potentials (SSVEPs) using covert visual attention (Lesenfants et al., 2013). In future applications, one alternative approach to realize an ssBCI might involve attention-modulated steady-state somatosensory evoked potentials (SSSEPs). The feasibility of such an ssBCI was already shown in healthy subjects (Pokorny et al., 2011). The usage of a tactile P300 BCI system with patients is explained in more detail in the next section. To conclude, initial results presented here, as well as the results of other similar studies (Goldfine et al., 2011; Cruse et al., 2011, indicate that some DOC patients could communicate their intent using EEG.

\section{COMMERCIAL SYSTEMS: MINDBEAGLE}

Patients normally stay in a rehabilitation institution for several months before they are released to professional home-care, a home for handicapped people, or to a private home - often without professional or technical support. Hospitals typically have medical assessment and rehabilitation facilities in the same unit. In these institutions, it is advantageous to have a system that can assess the cognitive functions of the patients in frequent intervals to show trends and can allow patients 
to communicate if cognitive functions are still present. Notwithstanding the described caveats, emerging EEG-based methods can facilitate both diagnosis and communication. The EEG is inexpensive, portable, widely available and objective, and has been used in hospitals for many years. However, practical EEG systems for non-expert users in home settings are not common.

As noted in the section titled "Requirements for an EEG-based diagnostic battery for patients with DOC", interviews with experts indicated that the most important properties of an assessment and communication system are:

- Dependence on EEG only (due to problems with fMRI and other means);

- $\quad$ Fast and easy mounting of electrodes;

- Automatic data analysis and result presentation;

- Ease of use by medical / technical assistants;

- $\quad$ Support for a test battery to assess cognitive function;

- The capability to provide communication for the patient;

- Cost; and

- Prognostic value of results.

The key purpose of such an EEG system is to assess cognitive functions and establish an interface for communication. This was realized through (i) auditory evoked potentials (AEP), (ii) vibrotactile (VT) evoked potentials and (iii) motor imagery experimental protocols. Both the auditory and vibrotactile experiments are designed to elicit a P300 response similar to a P300 spelling device. A P300 spelling device is a BCI that presents different characters on the computer screen, which each flash individually or in certain patterns. The user is asked to concentrate on just one of these characters. Whenever this character flashes, a P300 component appears in the EEG data. After several repetitions, the signal-to-noise ratio increases to a level that the $\mathrm{BCI}$ can detect and thereby select the corresponding character. This allows paralyzed patients to communicate (see Kaufmann et al., 2013c for the most recent result in patients with severe motor paralysis).

However, P300 BCIs based on visual stimuli do not work with patients who have lost their vision. Auditory paradigms can also be implemented using a frequent stimulus with a certain frequency and an infrequent stimulus with another frequency (see above). The user is asked to count how many times the infrequent stimulus occurs. Like with the visual P300 speller, the infrequent stimuli also produce a $\mathrm{P} 300$ response in the EEG. The same principle can be used for vibrotactile stimulation if $e . g$. the right hand is frequently stimulated and the left hand is infrequently stimulated. The EEG will also exhibit a $\mathrm{P} 300$ if the user is paying attention to the infrequent stimuli. This auditory and vibrotactile setup can assess whether the patient is able to follow instructions and in an experimental procedure (Kaufmann et al., 2013b). To answer yes and no questions, it is necessary to extend the vibrotactile setup to 3 stimulators. One of the stimulators applies the frequent stimuli, and 2 stimulators apply the infrequent stimuli. The user can concentrate on one of the infrequent stimulators to say (in this case) yes or no. Typically, an evoked potential is calculated by averaging the frequent and infrequent stimuli. A statistical analysis helps to visualize statistically significant differences, which is especially important for patient data collected in field settings, which is frequently noisy.

As noted above, motor imagery may also be used to control a BCI. In this case, the patient has to imagine a right or left hand movement for twofour seconds. This will result in an event-related desynchronization (ERD) and event-related synchronization (ERS) over the sensorimotor regions. Synchronization with the EEG data is necessary to avoid delays and jitters. The BCI system analyzes the acquired EEG data in real-time by implementing source derivation, pre-processing, parameter estimation and classification algorithms. Finally, 
Figure 6. Left, top: mindBEAGLE system with EEG cap, active EEG electrodes, g.USBamp amplifier, vibrotactile stimulator, in-ear headphones and a laptop computer running the software. Left, bottom: vibrotactile stimulators on the left and right hand. Right, top: BCI accuracy trend over several days. Right, bottom: EP of electrode position $C z$ with statistical test between target and non-target auditory stimuli. The EP shows a significant $P 300$ response. The bottom curve shows BCI accuracy over a number of target stimuli.
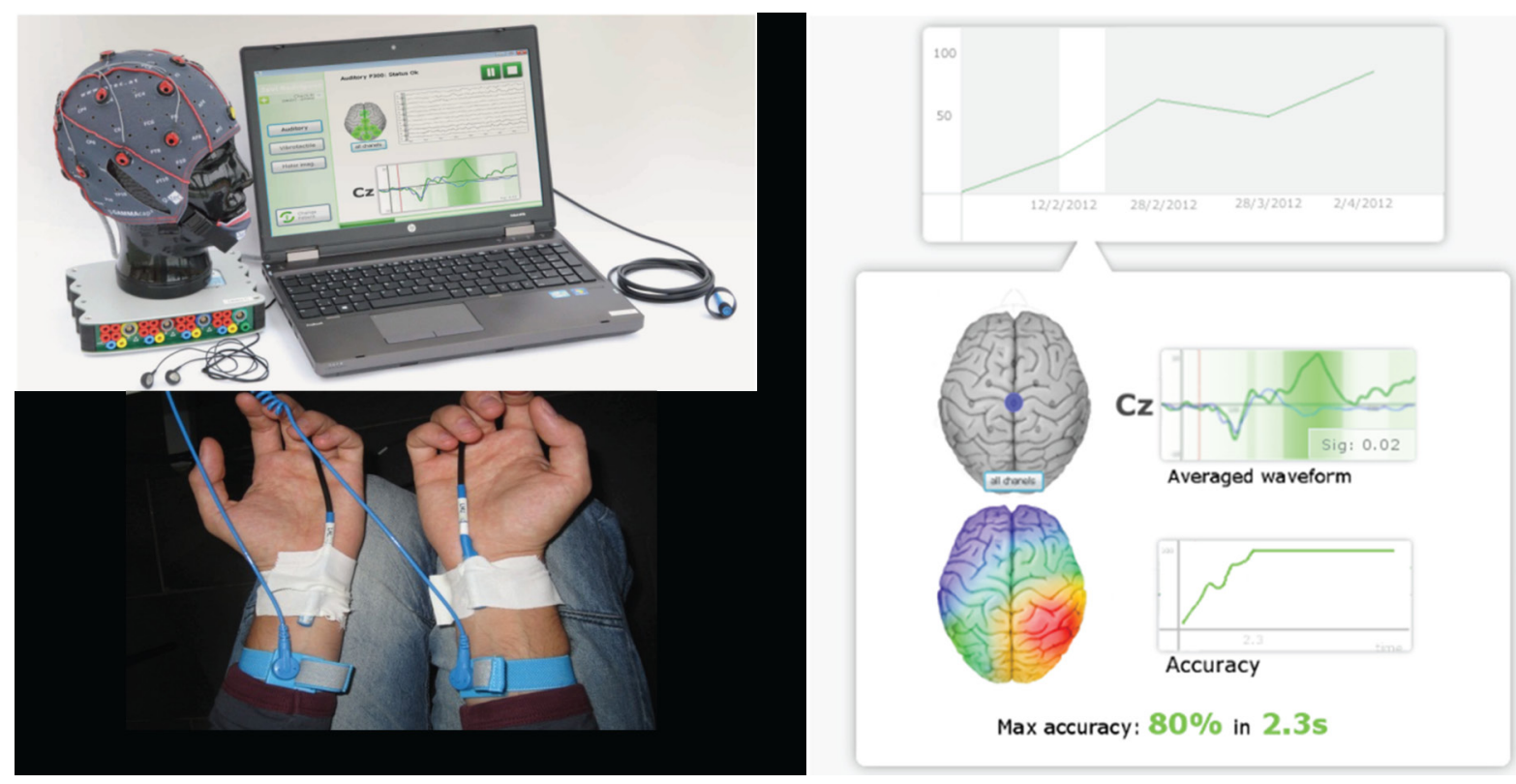

the BCI system decides whether the user imagined left or right hand movement, which can be used as a control signal to answer yes and no questions. If several repetitions are performed, the BCI system can calculate a mean accuracy that shows if the user is able to follow instructions. With two choices, such as yes or no, $50 \%$ accuracy would correspond to random classification.

The mindBEAGLE system (see Figure 6, left side) contains all these features and consists of an EEG amplifier with 16 channels that uses a 24 Bit analog to digital converter for high precision. The amplifier samples the EEG data at $256 \mathrm{~Hz}$ and sends the data via USB to the laptop computer system. Active EEG electrodes are used to get a robust EEG recording and avoid skin abrasion. They eliminate most of the artifacts from cable movements and power line interference. Therefore, high quality EEG recording can be performed even in an unshielded room. Sixteen active electrodes can be mounted with conductive electrode gel, normally within about 2 minutes, including ground and reference electrodes. The reference is mounted on the left ear lobe and the ground electrode is located on the forehead.

The laptop controls the data acquisition, experimental paradigm, signal processing and result presentation. To provide auditory stimulation, the laptop also has an internal audio interface which can stimulate the left and right ear via inear headphones and sends a trigger signal to the signal processing module for synchronization. For the vibrotactile stimulation, the system uses stimulators like those shown in Figure 6, left side. In this case, one stimulator is mounted on each wrist, and switched on to stimulate the hand for several hundred milliseconds. Both are connected via USB to the computer and are controlled in 
real-time through the experimental paradigm. For the motor imagery experiment, auditory instructions like "move the left hand" or "move the right hand" are given via the internal sound board. Perfect synchronization between the EEG data and auditory and tactile stimuli is critical to avoid delays or jitters.

The right side of Figure 6 shows the results for the auditory stimulation paradigm, using the evoked potential for electrode $\mathrm{Cz}$ on the vertex as an example. The Event-Related Potential (ERP) consists of the non-target averages (blue line) and of the target average (green line) from $100 \mathrm{~ms}$ before the stimulus onset until $700 \mathrm{~ms}$ post-stimulus. The green area around $250-350 \mathrm{~ms}$ indicates the statistically significant P300 response for this electrode with $\mathrm{p}<0.02$. The accuracy plot reaches $100 \%$ accuracy after 5 target stimuli, which shows that the BCI system can perfectly discriminate the P300 response provided a sufficient number of trials. This resulting accuracy is also logged into a trend analysis to assess the patient's P300 response over time.

The results for the vibrotactile experiment with 2 stimulators are similar to the results from the auditory experiment. The ERP waveform and BCI accuracy can be used to interpret the results. To answer questions in the vibrotactile paradigm, the subject is equipped with stimulators on each hand and the neck. Each of the stimulators is switched on for $100 \mathrm{~ms}$ with a short break in between. The subject is asked to focus on one of the stimulators and to count how many times it vibrates. Then, a caregiver asks a question and starts the paradigm. Like intendiX (www.intendix.com) or other visual P300 spellers, this elicits a P300 for the target stimulator, as shown in Figure 7, which allows the system to make a "yes" decision. Another kind of yes/no control approach was implemented with the motor imagery based BCI system, in which left or right movement imagery corresponded to "yes" or "no".

In summary, mindBEAGLE can assess auditory and vibrotactile P300 and motor imagery responses, which can help identify whether the patient is able to follow instructions. If a P300 response is detected with the statistical ERP analysis or with the BCI algorithms, then it is very likely that the patient can understand instructions and concentrate on the task. The same is the case for the motor imagery paradigm. The trend analysis helps to identify fluctuations and helps to optimize treatment. Since many patients have impaired vision, the motor imagery, auditory and vibrotactile paradigms are better suited to their abilities, and can also produce reliable results. However, these results must be confirmed with more DOC patients.

After the initial assessment with a positive P300 response, the patient can also be trained to use the system for communication. Using the auditory experiment and 2 stimulators, the patient

Figure 7. Vibrotactile P300 communication via stimulators placed on the left hand, right hand and neck (left, middle, and right panels, respectively). The middle panel shows a clear P300 when the subject attends to the right hand stimulator.

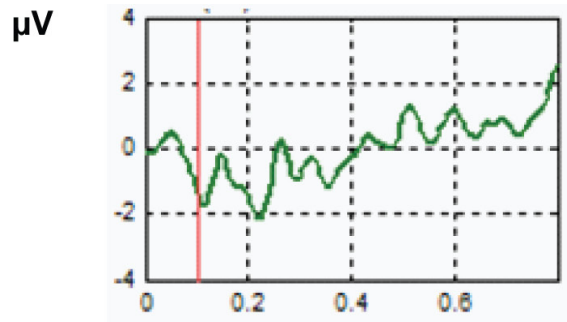

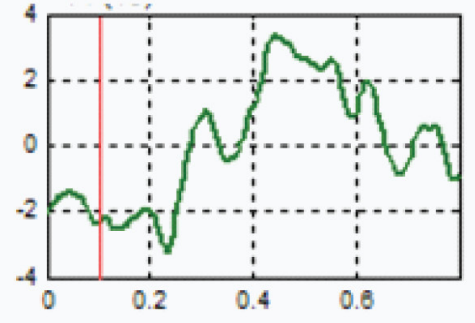

Time in seconds

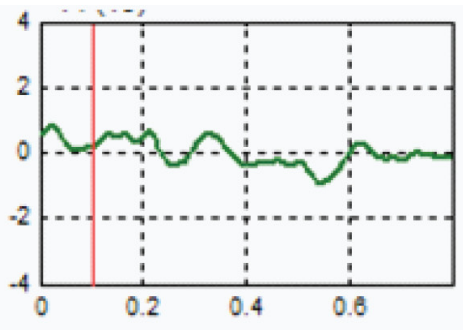


can simply generate a click. With 3 stimulators and the motor imagery experiment, the patient can even answer yes and no questions, and thus the mindBEAGLE provides an ssBCI as described above that can be used with DOC patients.

Hence, mindBEAGLE is a new, practical EEG based system that can work even in noisy, realworld environments. This section and the previous section about single-switch BCIs showed that new, EEG-based solutions can address some of the problems inherent in conventionalEEG-based tools for diagnosis and treatment. Next, we continue our discussion of challenges and potential solutions by considering a different tool to measure brain function in DOC patients.

\section{FUNCTIONAL NEAR-INFRARED SPECTROSCOPY (fNIRS): ANOTHER EMERGING SOLUTION FOR COMMUNICATION IN DOC PATIENTS?}

\section{Background}

Compared to the EEG or fMRI, fNIRS is a relatively new and rarely applied non-invasive functional brain imaging method. It measures a hemodynamic brain signal, similar to fMRI (Irani et al., 2007; Villringer \& Chance, 1997), and also shows similar vascular sensitivity (Huppert et al., 2006). Like fMRI, fNIRS relies on the neurovascular coupling and, thus, only indirectly measures local brain activation through hemodynamic changes that accompany changes in neuronal activity. In terms of signal acquisition, however, fMRI and fNIRS methods differ considerably.

Functional NIRS is based on the penetrability of biological tissue by near-infrared light (composed of wavelength of 650-950 nm). Emitter optodes (sources) that are placed on the head send out light that passes through the skull and the brain. On its way through the brain, the chromophores of oxygenated (oxy- $\mathrm{Hb}$ ) and deoxygenated hemoglobin (deoxy-Hb) within the blood vessels absorb the light according to their particular optical properties (Fallgatter et al., 2004; Irani et al., 2007).The remaining (reflected) light is picked up by detector optodes (detectors) placed on the head surface within a few centimeters from the sources. As oxy- and deoxy-Hb differ with respect to their absorption spectra (i.e., they absorb light of particular wavelengths), one can then calculate the relative concentrations for both of them separately from the amount of light attenuation (the ratio of emitted to reflected light) by using a modified Beer-Lambert law (Cope \& Delpy, 1988; Cope et al., 1988). Based on these concentrations, one can infer the functional state of neuronal tissue ('active' or 'non-active'). However, because of many uncontrollable influences, the absolute concentrations of the chromophores cannot be measured. Therefore, the exact brain activation level cannot be determined (Fallgatter et al., 2004). Nevertheless, relative changes in the signal time course are sufficient for localizing brain functions or for BCI applications, similar to fMRI.

Compared to fMRI, the main advantages of fNIRS are portability and reduced sensitivity to head movement artifacts. This makes fNIRS technology usable at the patients' bedside (for diagnostic purposes) or even beyond clinical settings in daily-life situations, e.g. for motor-independent communication. Moreover, fNIRS is relatively affordable, less technically demanding, and easier to operate. This might allow larger patient populations to benefit from hemodynamically based BCI applications. Furthermore, it does not require exposing patients to a strong magnetic field, and hence can be used close to paramagnetic medical equipment. Another advantage of fNIRS is that it is nearly silent. Thus, fNIRS can be regarded not only as a completely safe and harmless but also relatively comfortable method. While fNIRS has these benefits over fMRI, it only allows reliably measuring hemodynamic responses in cortical 
tissue that is close to the head surface (up to approximately $3 \mathrm{~cm}$ in depth). Thus, brain activation in, e.g., subcortical structures cannot be measured that would be accessible with fMRI. Moreover, the spatial resolution of fNIRS (in the range of a few cubic centimeters) is considerably lower than the resolution obtained with fMRI (in the range of a few cubic millimeters).

While fNIRS has been applied in fundamental neuroscience since the early 1990s, it has only recently been recognized as a potential BCI measurement technology (Coyle et al., 2004; 2007; Naito et al., 2007; Sitaram, et al., 2007; Luu\& Chau, 2009; Power et al., 2012). The following fNIRS study, performed in the context of the DECODER project, further explored the suitability of fNIRS for brain-computer interfacing.

\section{Methods}

Participants. Twelve healthy participants (age: 29.8 years \pm 9.6 ; five male, one left-handed) were included in the study that was approved by the local ethics committee of the Faculty of Psychology and Neuroscience at Maastricht University. All participants gave written informed consent.

General procedure, preparation and instruction. Seven mental tasks (mental calculation, mental drawing, mental rotation, mental singing, mental talking, spatial navigation and tennis imagery) were explained to the participants using standardized instructions. After ensuring that the participants understood each mental task, they were asked to choose the two mental tasks from the set that they thought are most easy and comfortable to perform. Participants had to undergo two identical functional runs. In each of these runs, they had to perform the two selected mental tasks nine times - three times for $5 \mathrm{~s}, 10 \mathrm{~s}$, and $15 \mathrm{~s}$ each. The two conditions alternated with resting phases of 20s in between. Participants were seated comfortably in a quiet room in front of a computer screen. They were instructed to engage in the mental task that was displayed on the screen as immediately and continuously as possible, and to stop as soon as the resting instruction was indicated. During the resting phase, participants should not engage in a specific thought or task. Only the first three letters of the second part of the mental task's name were displayed on the screen (e.g., "dra" instead of "mental drawing") to avoid a visual stimulation that could automatically evoke task-specific brain activation (Nachev \& Husain, 2007). For the same reason, the instructions were presented for only 2 s followed by a fixation cross. Visual stimuli were presented using E-Prime 2.0 software (Schneider et al., 2002).

Data acquisition. fNIRS data were recorded with a NIRScout 816 upgraded to 16 sources and 24 detectors (NIRx Medizintechnik GmbH, Berlin, Germany). The data were recorded for $9 \min 20 \mathrm{~s}$ for both $760 \mathrm{~nm}$ and $850 \mathrm{~nm}$ with a sampling rate of about $3.5 \mathrm{~Hz}$. Because of the limited number of sources and detectors, a montage with high resolution was only feasible for a confined area of the scalp. The montage shown in Figure 8A was used in order to get high-resolution data for the left hemisphere.

Data analysis. fNIRS data were analyzed using Satori (v0.8, Brain Innovation B.V., Maastricht, The Netherlands). T-values for each channel for the oxy- and deoxy-Hb time courses were computed and used as features for a linear support vector machine (SVM). The first run's data were used to 'train' and the second run's data were used to 'test' the SVM classifier. The classification was repeated with the second run's data to 'train' and the first run's data to 'test' the classifier. For each participant, both of the obtained 2-task classification accuracies were averaged. Moreover, the mean 2-class classification accuracies obtained separately for the two different 'training'/'testing' data sets were calculated.

\section{Results}

Each of the suggested mental tasks was chosen by at least one participant. Spatial navigation (n 
Figure 8. Optode placement and classification results of the fNIRS experiment. Panel A shows a $2 D$ representation of the optodes' montage. 40 optode locations were chosen to achieve a high-resolution coverage of the left hemisphere. White dots represent sources (16) and black dots represent detectors (24). Optrode positions are shown according to the extended 10-20 system of EEG electrode placement (Oostenveld \& Praamstra, 2001). Panel B displays the mean classification accuracies obtained separately for the two different 'training'/'testing' data sets and their mean classification accuracy. Error bars indicate variance across participants ( \pm standard error of mean -s.e.m.). Panel C shows the mental tasks selected and the achieved 2-task classification results for each participant. Moreover, the 2-task classification accuracy across all participants is displayed. Abbreviations/remarks: MC, mental calculation; $M D$, mental drawing; $M R$, mental rotation; $M S$, mental singing; MT, mental talking; SN, spatial navigation; TI, tennis imagery; error bars indicate variance across participants ( \pm s.e.m.).
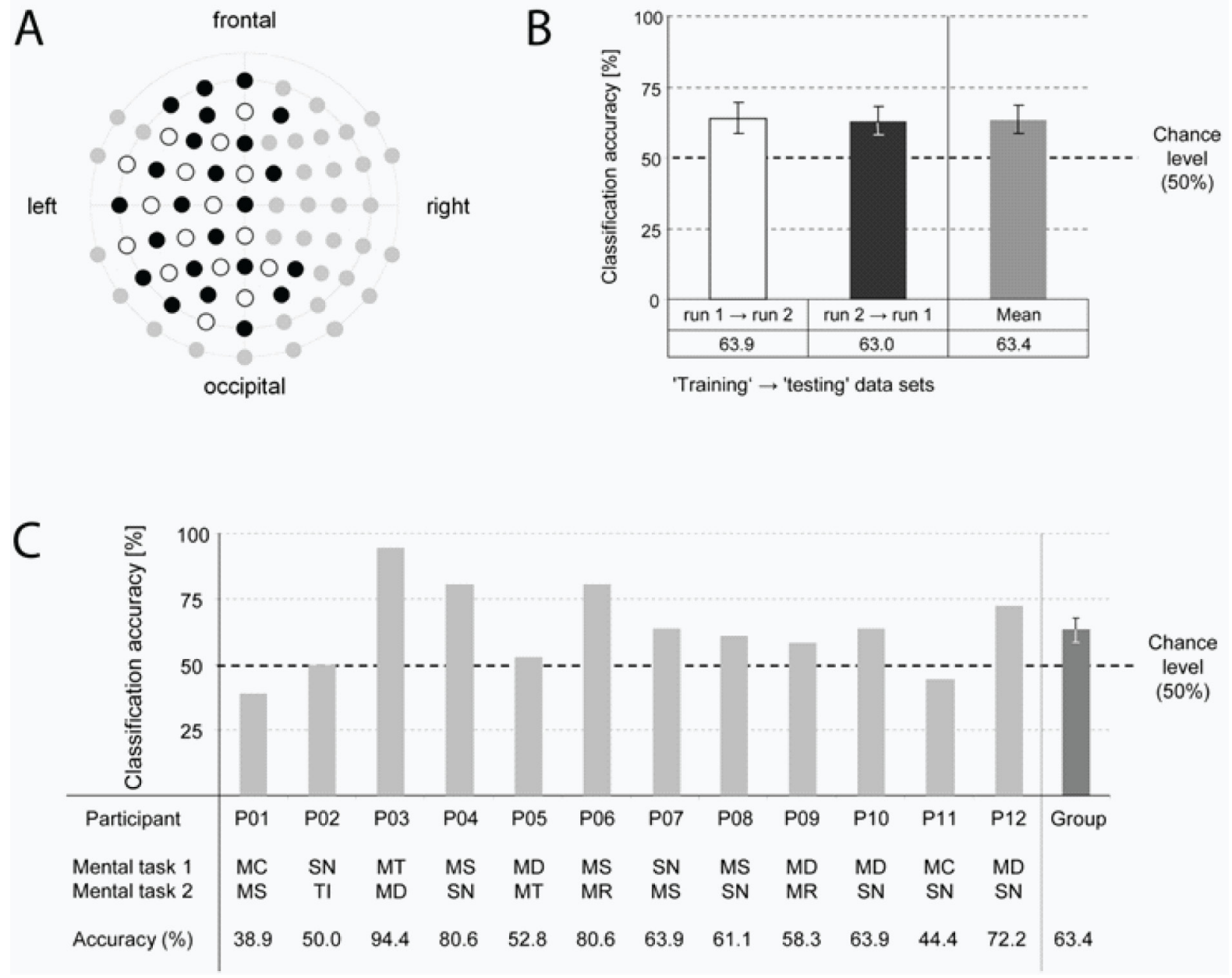

$=7)$ and mental drawing $(\mathrm{n}=5)$ were generally preferred, whereas tennis imagery was selected by only one participant (Figure 8C).

Averaged across participants, the achieved 2-task classification accuracy was $63.4 \%$ (chance level being $50 \%$ for differentiating between two mental tasks), which varied considerably between 38.9\% (P01) and 94.4\% (P03) (Figure 8C). The mean 2-task classification accuracies obtained 
separately for the two different 'training'/'testing' data sets only minimally differed (Figure 8B).

\section{Discussion}

In two thirds of the cases, the achieved individual 2 -task classification accuracies were above $60 \%$. However, it is critical to note that the obtained classification results varied considerably across participants. The high classification accuracy obtained for participant $\mathrm{P} 03$ indicates that, in principle, high classification performances can be achieved. Note that this participant was highly trained with regards to the mental tasks employed, whereas most other participants performed the mental tasks for the first time. This indicates that classification accuracies might considerably increase with intensive pre-training of the mental tasks, which might not be feasible when using fNIRS for assessing the level of consciousness, but should be possible in the context of motorindependent communication with BCI.

\section{Future Directions}

The aforementioned main limitations of fNIRS (relatively low spatial resolution, restricted depth pervasion) require a careful exploration of suited mental tasks that evoke differential brain activation patterns within superficial cortex regions (i.e., in crowns of gyri). In this context, it might prove beneficial to exploit the high resolution of fMRI and to transfer gained information on the localization of brain function to the fNIRS technology. Thus, one idea would be to perform fMRI and fNIRS experiments in the same individuals - preferably simultaneously. In this context, the individual information gained from fMRI experiments could be further used to optimize ('guide') optode placement and therewith increase the sensitivity of fNIRS. Additionally, more training data should be obtained, which will most likely increase classification accuracies. Moreover, fNIRS-based communication and control tools should be designed in a patient-tailored manner (e.g., individually selecting mental tasks) to optimize the separability of fNIRS brain activation patterns for each individual case and to make the device more comfortable. Finally, researchers should make a strong effort to explain the considerable inter-individual variability. The suggested combined fMRI and fNIRS measurements in the same individual may help in the future to better understand the observed inter-subject variability.

Taking into account the current state-of-the-art and the resulting opportunities for further development, we consider fNIRS-based brain-computer interfacing a promising technology with a clear potential for motor-independent communication.

\section{CONCLUSION}

Conventional methods for assessing the level of consciousness in patients with DOC and providing communication are insufficient. Both interviews with clinicians and numerous imaging studies have shown that misdiagnoses are common, even in hospital settings with well-established procedures. Furthermore, tools that can allow for communication are promising but require further development. Several advancements within the DECODER project and other efforts have further elucidated conventional problems and possible solutions, and have established foundations for improved portable systems that may rely on EEG and/or fNIRS. Different possible future directions were discussed within respective sections, supporting the overall conclusion that new, BCI-based technologies are advancing rapidly and are likely to 
provide improved diagnosis and communication for patients with disorders of consciousness or in the locked-in state.

The issue of BCIs used in DOC patients may be regarded as key for further clinical practice, but there is relatively little work analyzing functional neuroimaging data in DOC patients. Our understanding of higher cognitive processes and related brain activity is too limited to develop thorough and precise methods to understand DOC patient data, and research such as in the DECODER project might help to change this situation. By developing BCI technology to assess, understand, and communicate with DOC patients, we might not only change the lives of these patients and their families and caregivers, but also solve some scientific, clinical and ethical problems. Thus, results and conclusions of the DECODER project provide a basis for improved guidelines and new clinical and research procedures. In the near future, BCI based systems for assessment and communication will become available that will allow us to use BCI technology with more patients and perform further scientific studies.

\section{REFERENCES}

Allison, B. Z., Dunne, S., Leeb, R., Millan, J., \& Nijholt, A. (2013). Recent and upcoming BCI progress: Overview, analysis, and recommendations. In Towards Practical BCIs: Bridging the Gap from Research to Real-World Applications. Springer-Verlag.

American Congress of Rehabilitation Medecine. (1995). Recommendation for use of uniform nomenclature pertinent to patients with severe alterations of consciousness. Arch Phys Med Rehabil 76, 205-209.
Bardin, J. C., Fins, J. J., \& Katz, D. I. et al. (2011). Dissociations between behavioural and functional magnetic resonance imaging-based evaluations of cognitive function after brain injury. Brain, 134, 769-782. doi:10.1093/brain/awr005

Bianchi, L., Quitadamo, L. R., Garreffa, G., Cardarilli, G. C., \& Marciani, M. G. (2007). Performances evaluation and optimization of brain computer interface systems in a copy spelling task. IEEE Transactions on Neural Systems and Rehabilitation Engineering, 15(2), 207-216. doi:10.1109/TNSRE.2007.897024

Billinger, M., Daly, I., Kaiser, V., Jin, J., Allison, B. Z., Müller-Putz, G. R., \& Brunner, C. (2013). Is it significant? Guidelines for reporting BCI performance. In Towards Practical BCIs: Bridging the Gap from Research to Real-World Applications. Springer-Verlag.

Blair, R. C., \& Karniski, W. (1993). An alternative method for significance testing of waveform difference potentials. Psychophysiology, 30(5), 518-524. doi:10.1111/j.1469-8986.1993. tb02075.x

Bodart, O., Laureys, S., \& Gosseries, O. (2013). Coma and disorders of consciousness: scientific advances and practical considerations for clinicians. Seminars in Neurology, 33(2), 83-90. doi:10.1055/s-0033-1348965

Boly, M., Coleman, M. R., \& Davis, M. H. et al. (2007). When thoughts become action: an fMRI paradigm to study volitional brain activity in non-communicative brain injured patients. $\mathrm{Neu}$ roImage, 36, 979-992. doi:10.1016/j.neuroimage.2007.02.047

Bostanov, V., \& Kotchoubey, B. (2006). The tCWT: a new ERP detection and quantification method based on the continuous wavelet transform and Student's t-statistics. Clinical Neurophysiology, 117(12), 2627-2644. doi:10.1016/j. clinph.2006.08.012 
Brownson, R. C., Allen, P., Duggan, K., Stamatakis, K. A., \& Erwin, P. C. (2012). Fostering more-effective public health by identifying administrative evidence-based practices: A review of the literature. American Journal of Preventive Medicine, 43(3), 309-319. doi:10.1016/j. amepre.2012.06.006

Bruno, M. A., Vanhaudenhuyse, A., Thibaut, A., Moonen, G., \& Laureys, S. (2011). From unresponsive wakefulness to minimally conscious PLUS and functional locked-in syndromes: recent advances in our understanding of disorders of consciousness. Journal of Neurology, 258(7), 1373-1384. doi:10.1007/s00415-011-6114-x

Caria, A., Sitaram, R., \& Birbaumer, N. (2012). Real-time fMRI: A tool for local brain regulation. The Neuroscientist, 18(5), 487-501. doi:10.1177/1073858411407205

Chadwick, M. J., Hassabis, D., Weiskopf, N., \& Maguire, E. A. (2010). Decoding individual episodicmemory traces in the human hippocampus. Current Biology, 20, 544-547. doi:10.1016/j. cub.2010.01.053

Coles, M. G., Gratton, G., \& Donchin, E. (1988). Detecting early communication: using measures of movement-related potentials to illuminate human information processing. Biological Psychology, 26(1-3), 69-89. doi:10.1016/03010511(88)90014-2

Connolly, J. F., Phillips, N. A., Stewart, S. H., \& Brake, W. G. (1992). Event-related potential sensitivity to acoustic and semantic properties of terminal words in sentences. Brain and Language, 43(1), 1-18. doi:10.1016/0093-934X(92)90018-A
Cope, M., \& Delpy, D. T. (1988). System for long-term measurement of cerebral blood and tissue oxygenation on newborn infants by near infra-red transillumination. Medical \& Biological Engineering \& Computing, 26(3), 289-294. doi:10.1007/BF02447083

Cope, M., Delpy, D. T., Reynolds, E. O., Wray, S., Wyatt, J., \& van der Zee, P. (1988). Methods of quantitating cerebral near infrared spectroscopy data. Advances in Experimental Medicine and Biology, 215, 183-189. doi:10.1007/978-1-46159510-6_21

Coyle, S., Ward, T., Markham, C., \& McDarby, G. (2004). On the suitability of near-infrared (NIR) systems for next-generation brain-computer interfaces. Physiological Measurement, 25(4), 815-822. doi:10.1088/0967-3334/25/4/003

Coyle, S. M., Ward, T. E., \& Markham, C. M. (2007). Brain-computer interface using a simplified functional near-infrared spectroscopy system. Journal of Neural Engineering, 4(3), 219-226. doi:10.1088/1741-2560/4/3/007

Cruse, D. et al. (2011). Bedside detection of awareness in the vegetative state: A cohort study. Lancet, 378(9809), 2088-2094. doi:10.1016/ S0140-6736(11)61224-5

D’Arcy, R. C. N., Ghosh-Hajra, S., Liu, C., Sculthorpe, L., \& Weaver, D. F. (2011). Towards brain first-aid: A diagnostic device for conscious awareness. IEEE Transactions on Bio-Medical Engineering, 58(3), 750-754. doi:10.1109/ TBME.2010.2090880

Daltrozzo, J., Wioland, N., Mutschler, V., \& Kotchoubey, B. (2007). Predicting outcome of coma using event-related brain potentials: A metaanalytic approach. Clinical Neurophysiology, 118, 606-614. doi:10.1016/j.clinph.2006.11.019 
deCharms, R. C. (2007). Reading and controlling human brain activation using real-time functional magnetic resonance imaging. Trends in Cognitive Sciences, 11, 473-481. doi:10.1016/j. tics.2007.08.014

deCharms, R. C. (2008). Applications of real-time fMRI.Nature Reviews. Neuroscience, 9, 720-729. doi:10.1038/nrn2414

Donchin, E. (1981). Surprise!? Surprise? Psychophysiology, 18(5), 493-513. doi:10.1111/j.1469-8986.1981.tb01815.x

Duffy, F. H., Burchfiel, J. L., \& Lombroso, C. T. (1979). Brain electrical activity mapping (BEAM), A method for extending the clinical utility of EEG and evoked potential data. Annals of Neurology, 5, 309-321. doi:10.1002/ana.410050402

Duncan, C. C., Barry, R. J., Connolly, J. F., Fischer, C., Michie, P. T., \& Naatanen, R. et al. (2009). Event-related potentials in clinical research: guidelines for eliciting, recording, and quantifying mismatch negativity, $\mathrm{P} 300$, and N400. Clinical Neurophysiology, 120(11), 1883-1908. doi:10.1016/j.clinph.2009.07.045

Duncan-Johnson, C. C., \& Donchin, E. (1977). On Quantifying Surprise: The Variation of Event-Related potentials With Subjective Probability. Psychophysiology, 14(5), 456-467. doi:10.1111/j.1469-8986.1977.tb01312.x

Erlbeck, H., \& Kübler, A. (2013). Requirements for a new EEG-based diagnostic battery for the detection of the level of consciousness. Paper presented at the 4th Workshop on Tools for Brain Computer Interaction (TOBI). Sion, Switzerland.

Fallgatter, A. J., Ehlis, A., Wagener, A., Michel, T., \& Herrmann, M. J. (2004). Near-infrared spectroscopy in psychiatry. Der Nervenarzt, 75(9), 911-916. doi:10.1007/s00115-002-1457-2
Fischer, C., Morlet, D., Bouchet, P., Luaute, J., Jourdan, C., \& Salord, F. (1999). Mismatch negativity and late auditory evoked potentials in comatose patients. ClinicalNeurophysiology, 110, 1601-1610. doi:10.1016/S1388-2457(99)001315

Fischler, I., Jin, Y. S., Boaz, T. L., Perry, N. W., \& Childers, D. G. (1987). Brain potentials related to seeing one's own name. Brain and Language, 30, 245-262. doi:10.1016/0093-934X(87)90101-5

Friedrich, E. V. C., Scherer, R., \& Neuper, C. (2012). The effect of distinct mental strategies on classification performance for braincomputer interfaces. International Journal of Psychophysiology, 84(1), 86-94. doi:10.1016/j. ijpsycho.2012.01.014

Friston, K. J., Holmes, A. P., \& Poline, J. B. et al. (1995). Analysis of fMRI time-series revisited. NeuroImage, 2, 45-53. doi:10.1006/ nimg.1995.1007

Giacino, J. T., Ashwal, S., Childs, N., Cranford, R., Jennett, B., \& Katz, D. I. et al. (2002). The minimally conscious state: Definition and diagnostic criteria. Neurology, 58, 349-353. doi:10.1212/ WNL.58.3.349

Giacino, J. T., Kalmar, K., \& Whyte, J. (2004). The JFK Coma Recovery Scale-Revised: Measurement Characteristics and Diagnostic Utility. Archives of Physical Medicine and Rehabilitation, 85, 2020-2029. doi:10.1016/j.apmr.2004.02.033

Goldfine, A. M., Victor, J. D., Conte, M. M., Bardin, J. C., \& Schiff, N. D. (2011). Determination of awareness in patients with severe brain injury using EEG power spectral analysis. Clinical Neurophysiology, 122(11),2157-2168. doi:10.1016/j. clinph.2011.03.022 
Groppe, D. M., Urbach, T. P., \& Kutas, M. (2011). Mass univariate analysis of event-related brain potentials/fields I: A critical tutorial review. Psychophysiology, 48(12), 1711-1725. doi:10.1111/j.1469-8986.2011.01273.x

Gsell, W., De Sadeleer, C., \& Marchalant, Y. et al. (2000). The use of cerebral blood flow as an index of neuronal activity in functional neuroimaging: experimental and pathophysiological considerations. Journal of Chemical Neuroanatomy, 20, 215-224. doi:10.1016/S0891-0618(00)00095-8

Guérit, J. M. (2005). Neurophysiological patterns of vegetative and minimally conscious states. $\mathrm{Neu}$ ropsychological Rehabilitation, 15(3-4), 357-371. doi:10.1080/09602010443000560

Guger, C., Krausz, G., Allison, B. Z., \& Edlinger, G. (2012). A comparison of dry and gel-based electrodes for P300 BCIs. Frontiers in Neuroscience, 6, 60. doi:10.3389/fnins.2012.00060

Guthrie, D., \& Buchwald, J. S. (1991). Significance testing of difference potentials. Psychophysiology, 28(2), 240-244. doi:10.1111/j.1469-8986.1991. tb00417.x

Hagoort, P. (2005). On Broca, brain, and binding: a new framework. Trends in Cognitive Sciences, 9(9), 416-423. doi:10.1016/j.tics.2005.07.004

Hagoort, P., Brown, C. M., \& Swaab, T. Y. (1996). Lexical-semantic event-related potential effects in patients with left hemisphere lesions and aphasia, and patients with right hemisphere lesions without aphasia. Brain, 119(2), 627-649. doi:10.1093/ brain/119.2.627

Haynes, J. D., \& Rees, G. (2006). Decoding mental states from brain activity in humans. Nature Reviews. Neuroscience, 7,523-534. doi:10.1038/ nrn1931
Healy, J. (2010). The vegetative state: Life, death and consciousness. Journal of the Intensive Care Society, 11, 118-123.

Herbert, C., \& Kübler, A. (2011). Dogs cannot bark: event-related brain responses to true and false negated statements as indicators of higher-order conscious processing. PLoS ONE, 6(10), e25574. doi:10.1371/journal.pone.0025574

Holz, E., Kaufmann, T., Desidiri, L., Malavasi, M., Hoogerwerf, E. J., \& Kübler, A. (2013). User Centred Design in BCI Development. In Towards Practical Brain-Computer Interfaces: Bridging the Gap from Research to Real-World Applications. Berlin: Springer-Verlag.

Horki, P., Pokorny, C., Klobassa, D., Pichler, G., \& Müller-Putz, G. (2012). Detection of mental imagery and attempted movement in patients with disorders of consciousness using EEG. Paper presented at BBCI Workshop 2012 on Advances in Neurotechnology. Berlin, Germany.

Huppert, T. J., Hoge, R. D., Diamond, S. G., Franceschini, M. A., \& Boas, D. A. (2006). A temporal comparison of BOLD, ASL, and NIRS hemodynamic responses to motor stimuli in adult humans. NeuroImage, 29(2), 368-382. doi:10.1016/j.neuroimage.2005.08.065

Irani, F., Platek, S. M., Bunce, S., Ruocco, A. C., $\&$ Chute, D. (2007). Functional near infrared spectroscopy (fNIRS), an emerging neuroimaging technology with important applications for the study of brain disorders. The Clinical Neuropsychologist, 21(1), 9-37. doi:10.1080/13854040600910018

Jacobsen, T., \& Schröger, E. (2003). Measuring duration mismatch negativity. Clinical Neurophysiology, 114(6), 1133-1143. doi:10.1016/ S1388-2457(03)00043-9 
Kane, N. M., Butler, S. R., \& Simpson, T. (2000). Coma outcome prediction using event related potentials: P3 and mismatch negativity. Audiology \& Neuro-Otology, 5, 186-191. doi:10.1159/000013879

Kaufmann, T., Holz, E., \& Kübler, A. (2013a). The importance of user-centered design in BCI development: A case study with a locked-in patient. In Proceedings of the 4th Workshop on Tools for Brain Computer Interaction (TOBI). Sion, Switzerland: TOBI.

Kaufmann, T., Holz, E. M., \& Kübler, A. (2013b). Comparison of tactile, auditory, and visual modality for brain-computer interface use: a case study with a patient in the locked-in state. Front. Neurosci., 7, 129. doi:10.3389/fnins.2013.00129

Kaufmann, T., Schulz, S. M., Köblitz, A., Renner, G., Wessig, C., \& Kübler, A. (2013c). Face stimuli effectively prevent brain-computer interface inefficiency in patients with neurodegenerative disease. Clinical Neurophysiology, 124(5), 893-900. doi:10.1016/j.clinph.2012.11.006

Kay, K. N., Naselaris, T., Prenger, R. J., \& Gallant, J. L. (2008). Identifying natural images from human brain activity. Nature, 452, 352-355. doi:10.1038/nature06713

Kotchoubey, B. (2005). Apallic syndrome is not apallic: Is vegetative state vegetative? Neuropsychological Rehabilitation, 15(3-4), 333-356. doi:10.1080/09602010443000416

Kotchoubey, B., \& Lang, S. (2001). Event-related potentials in an auditory semantic oddball task in humans. Neuroscience Letters, 310, 93-96. doi:10.1016/S0304-3940(01)02057-2

Kotchoubey, B., Lang, S., Bostanov, V., \& Birbaumer, N. (2002). Is there a Mind? Electrophysiology of Unconscious Patients. Physiology (Bethesda, MD), 17, 38-42.
Kotchoubey, B., Lang, S., Herb, E., Maurer, P., Schmalohr, D., Bostanov, V., \& Birbaumer, N. (2003). Stimulus complexity enhances auditory discrimination in patients with extremely severe brain injuries. Neuroscience Letters, 352, 129-132. doi:10.1016/j.neulet.2003.08.045

Kotchoubey, B., Lang, S., Mezger, G., Schmalohr, D., Schneck, M., \& Semmler, A. et al. (2005). Information processing in severe disorders of consciousness: Vegetative state and minimally conscious state. Clinical Neurophysiology, 116(10), 2441-2453. doi:10.1016/j.clinph.2005.03.028

Kübler, A., Holz, E., \& Kaufmann, T. (in press). A user centred approach for bringing BCI controlled applications to end-users. Brain-Computer Interface.

Kübler, A., \& Kotchoubey, B. (2007). Braincomputer interfaces in the continuum of consciousness. Current Opinion in Neurology, 20, 643-649. doi:10.1097/WCO.0b013e3282f14782

Kutas, M., \& Federmeier, K. D. (2011). Thirty years and counting: Finding meaning in the N400 component of the event-related brain potential (ERP). Annual Review of Psychology, 62, 621647. doi:10.1146/annurev.psych.093008.131123

Kutas, M., \& Hillyard, S. A. (1980). Reading senseless sentences: Brain potentials reflect semantic incongruity. Science, 207, 203-205. doi:10.1126/ science. 7350657

Kwong, K. K., Belliveau, J. W., \& Chesler, D. A. et al. (1992). Dynamic magnetic resonance imaging of human brain activity during primary sensory stimulation. Proceedings of the National Academy of Sciences of the United States of America, 89, 5675-5679. doi:10.1073/pnas.89.12.5675

LaConte, S. M., Peltier, S. J., \& Hu, X. P. (2007). Real-time fMRI using brain-state classification. Human Brain Mapping, 28, 1033-1044. doi:10.1002/hbm.20326 
Lang, S., \& Kotchoubey, B. (2002). Brain responses to number sequences with and without active task requirement. Clinical Neurophysiology, 113, 1734-1741. doi:10.1016/S13882457(02)00267-5

Lang, S., Kotchoubey, B., Lutz, A., \& Birbaumer, N. (1997). Was tut man, wenn man nichts tut? Kognitive EKP-Komponenten ohne kognitive Aufgabe.Zeitschrift für Experimentelle Psychologie, 44(1), 138-162.

Laureys, S., Celesia, G. G., Cohadon, F., Lavrijsen, J., León-Carrión, J., \& Sannita, W. G. et al. (2010). European Task Force on Disorders of Consciousness: Unresponsive wakefulness syndrome: a new name for the vegetative state or apallic syndrome. BMC Medicine, 1, 8-68. doi: doi:10.1186/1741-7015-8-68

Lee, J. H., Marzelli, M., Jolesz, F. A., \& Yoo, S. S. (2009). Automated classification of fMRI data employing trial-based imagery tasks. Medical Image Analysis, 13, 392-404. doi:10.1016/j. media.2009.01.001

Lesenfants, D. et al. (2013). Towards independent SSVEP-based brain-computer interface. IEEE Transactions on Neural Systems and Rehabilitation Engineering.

Luck, S. J. (2005). An introduction to the eventrelated potential technique. Cambridge, MA: MIT Press.

Luu, S., \& Chau, T. (2009). Decoding subjective preference from single-trial near-infrared spectroscopy signals. Journal of Neural Engineering, 6(1),016003. doi:10.1088/1741-2560/6/1/016003

Mallat, S. (2007). A wavelet tour of signal processing (2nd ed.). San Diego, CA: Academic Press.

Monti, M. M., Pickard, J. D., \& Owen, A. M (2013). Visual Cognition in Disorders of Consciousness: from V1 to top-down attention. Human Brain Mapping, 34(6), 1245-1253. doi:10.1002/ hbm.21507
Monti, M. M., Vanhaudenhuyse, A., \& Coleman, M. R. et al. (2010). Willful modulation of brain activity in disorders of consciousness. The New England Journal of Medicine, 362, 579-589. doi:10.1056/NEJMoa0905370

Müller-Putz, G., et al. (2012). The auditory P300-based ssBCI: a door to minimally conscious patients? In Proceedings of the 34th Annual International IEEE EMBS Conference (pp. 1-4). San Diego, CA: IEEE.

Müller-Putz, G. R., Pokorny, C., Klobassa, D., \& Horki, P. (2013). A single switch BCI based on passive and imagined movements: Towards restoring communication in minimally conscious patients. International Journal of Neural Systems. doi:10.1142/S0129065712500372

Näätänen, R.,Paavilainen,P., Rinne, T., \& Alho, K. (2007). The mismatch negativity (MMN) in basic research of central auditory processing: a review. Clinical Neurophysiology, 118(12), 2544-2590. doi:10.1016/j.clinph.2007.04.026

Näätänen, R., Pakarinen, S., Rinne, T., \& Takegata, R. (2004). The mismatch negativity (MMN), towards the optimal paradigm. Clinical Neurophysiology, 115(1), 140-144. doi:10.1016/j. clinph.2003.04.001

Nachev, P., \& Husain, M. (2007). Comment on Detecting awareness in the vegetative state. Science, 315(5816), 1221. doi:10.1126/science.1135096

Naci, L., Monti, M. M., Cruse, D., Sorger, B., Rainer, G. A., \& Kotchoubey, B. et al. (2012). Brain computer interfaces for communication with non-responsive patients. Annals of Neurology, 72(3), 312-323. doi:10.1002/ana.23656

Naito, M., Michioka, Y., Ozawa, K., Ito, Y., Kiguchi, M., \& Kanazawa, T. (2007). A Communication Means for Totally Locked-in ALS Patients Based on Changes in Cerebral Blood Volume Measured with Near-Infrared Light. IEICE Trans Inf \& Syst. E (Norwalk, Conn.), 90-D(7), 1028-1037. 
Neumann, N., \& Kotchoubey, B. (2004). Assessment of cognitive functions in severely paralyzed and severely brain-damaged patients: Neuropsychological and electrophysiological techniques. Brain Research Protocols, 14, 25-36. doi:10.1016/j.brainresprot.2004.09.001

Ogawa, S., Menon, R. S., \& Tank, D. W. et al. (1993). Functional brain mapping by blood oxygenationlevel-dependent contrast magnetic resonance imaging: A comparison of signalcharacteristics with a biophysical model. Biophysical Journal, 64, 803-812. doi:10.1016/S00063495(93)81441-3

Oostenveld, R., \& Praamstra, P. (2001). The five percent electrode system for high-resolution EEG and ERP measurements. Clinical Neurophysiology, 112(4), 713-719.doi:10.1016/S13882457(00)00527-7

Owen, A. M., \& Coleman, M. R. (2008). Using neuroimaging to detect awareness in disorders of consciousness. Functional Neurology, 23, 189-194.

Owen, A. M., Coleman, M. R., \& Boly, M. et al. (2006). Detecting awareness in the vegetative state. Science,313, 1402.doi:10.1126/science.1130197

Owen, A. M., Coleman, M. R., Menon, D. K., Berry, E. M., Johnsrude, I. S., \& Rodd, J. M. et al. (2005). Using a hierarchical approach to investigate residual auditory cognition in persistent vegetative state. Progress in Brain Research, 150, 457-471. doi:10.1016/S0079-6123(05)50032-3

Patterson, J. R., \& Grabois, M. (1986). Locked-in syndrome: a review of 139 cases. Stroke, 17(4), 758-764. doi:10.1161/01.STR.17.4.758

Peterson, N. N., Schroeder, C. E., \& Arezzo, J. C. et al. (1995). Neural generators of early cortical somatosensory evoked potentials in the awake monkey. Electroencephalography and Clinical Neurophysiology, 96,248-260. doi:10.1016/01685597(95)00006-E
Picton, T. W., Bentin, S., Berg, E., Donchin, E., Hillyard, S. A., \& Johnson, R. et al. (2000). Guidelines for using human event-related potentials to study cognition: Recording standards and publication criteria. Psychophysiology, 37, 127-152. doi:10.1111/1469-8986.3720127

Plum, F., \& Posner, J. (1966). The diagnosis of stupor and coma. Philadelphia: Davis, F.A.

Pokorny, C., et al. (2013). The auditory P300based single-switch BCI: Paradigm transition from healthysubjects to minimally conscious patients. Artificial Intelligence in Medicine.

Pokorny, C., Breitwieser, C., Neuper, C., \& MüllerPutz, G. (2011). Towards a Single-Switch BCI Based on Steady-State Somatosensory Evoked Potentials. In Proceedings of the 5th International Brain-Computer Interface Conference 2011 (pp. 200-203). Graz, Austria: Academic Press.

Power, S. D., Kushki, A., \& Chau, T. (2012). Automatic single-trial discrimination of mental arithmetic, mental singing and the no-control state from prefrontal activity: Toward a threestate NIRS-BCI. BMC Research Notes, 5, 141. doi:10.1186/1756-0500-5-141

Real, R., Erlbeck, H., Veser, S., Kotchoubey, B., \& Kübler, A. (2013). Assessing information processing in patients with long term and severe disorders of consciousness. In Proceedings of the 5th International Brain-Computer Interface Meeting 2013 (pp. 36-37). Pacific Grove, CA: Academic Press.

Ricci, E., Haider, S., Vaughan, T. M., \& Hill, N. J. (2013). An improved auditory streaming BCI with voice stimuli. In Proceeding sf the 5th International Brain-Computer Interface Meeting 2013 (pp. 4-5). Pacific Grove, CA: Academic Press. 
Rossini, P. M., Altamura, C., \& Ferretti, A. et al. (2004). Does cerebrovascular disease affect the coupling between neuronal activity and local haemodynamics? Brain, 127, 99-110. doi:10.1093/ brain/awh012

Rousseaux, M., Castelnot, E., Rigaux, P., Kozlowski, O., \& Danze, F. (2009). Evidence of persisting cognitive impairment in a case series of patients with locked-in syndrome. Journal of Neurology, Neurosurgery, and Psychiatry, 80(2), 166-170. doi:10.1136/jnnp.2007.128686

Schnakers, C., Vanhaudenhuyse, A., Giacino, J., Ventura, M., Boly, M., \& Majerus, S. et al. (2009a). Detecting consciousness in a total locked-in syndrome: an active eventrelated paradigm. Neurocase, 15(4), 271-277. doi:10.1080/13554790902724904

Schnakers, C., Vanhaudenhuyse, A., Giacino, J., Ventura, M., Boly, M., \& Majerus, S. et al. (2009b). Diagnostic accuracy of the vegetative and minimally conscious state: Clinical consensus versus standardized neurobehavioral assessment. BMC Neurology, 9, 35. doi:10.1186/1471-2377-9-35

Schneider, W., Eschman, A., \& Zuccolotto, A. (2002). E-Prime Reference Guide. Pittsburgh, PA: Psychology Software Tools Inc.

Schröger, E., \& Wolff, C. (1996). Mismatch response of the human brain to changes in sound location. Neuroreport, 7, 3005-3008. doi:10.1097/00001756-199611250-00041

Sitaram, R., Lee, S., \& Ruiz, S. et al. (2011). Realtime support vector classification and feedback of multiple emotional brain states. NeuroImage, 56, 753-765. doi:10.1016/j.neuroimage.2010.08.007
Sitaram, R., Zhang, H., Guan, C., Thulasidas, M., Hoshi, Y., \& Ishikawa, A. et al. (2007). Temporal classification of multichannel nearinfrared spectroscopy signals of motor imagery for developing a brain-computer interface. NeuroImage, 34(4), 1416-1427. doi:10.1016/j. neuroimage.2006.11.005

Sitnikova, T., Salisbury, D. F., Kuperberg, G., \& Holcomb, P. I. (2002). Electrophysiological insights into language processing in schizophrenia. Psychophysiology, 39(6), 851-860. doi:10.1111/1469-8986.3960851

Villringer, A., \& Chance, B. (1997). Non-invasive optical spectroscopy and imaging of human brain function. Trends in Neurosciences, 20(10), 435-442. doi:10.1016/S0166-2236(97)01132-6

Wijnen, V. J., van Boxtel, G. J., Eilander, H. J., \& de Gelder, G. (2007). Mismatch negativity predicts recovery from the vegetative state. Clinical Neurophysiology, 118, 597-605. doi:10.1016/j. clinph.2006.11.020

Wolpaw, J. R., Birbaumer, N., McFarland, D. J., Pfurtscheller, G., \& Vaughan, T. M. (2002). Brain-computer interfaces for communication and control. Clinical Neurophysiology, 113,767-791. doi:10.1016/S1388-2457(02)00057-3

Wolpaw, J. R., \& Wolpaw, E. W. (2012). BrainComputer Interfaces: Principles and Practice. Oxford University Press.

Yuan, P., Gao, X., Allison, B. Z., Wang, Y., Bin, G., \& Gao, S. (2013). A study on existing problems of information transfer rate estimation in online brain-computer interfaces. Journal of Neural Engineering, 10, 026014. doi:10.1088/17412560/10/2/026014

Yvert, B., Bertrand, O., \& Pernier, J., \& Iimoniemi, R. J. (1998). Human cortical responses evoked by dichotically presented tones of different frequencies. Neuroreport, 9, 1115-1119. doi:10.1097/00001756-199804200-00029 
Zickler, C., Riccio, A., Leotta, F., HillianTress, S., Halder, S., \& Holz, E. et al. (2011). A Brain-Computer Interface as Input Channel for a Standard Assistive Technology Software. Clinical EEG and Neuroscience, 42, 236-244. doi:10.1177/155005941104200409

\section{KEY TERMS AND DEFINITIONS}

Auditory Evoked Potential (AEP): A category of EEG activity elicited by auditory stimuli, such as tones or words. Other sensory information can produce other evoked potentials, such as visual evoked potentials (VEPs) or somatosensory evoked potentials (SEPs).

Battery for the Assessment of the Level of Consciousness (BAC): A series of tests that may be used to evaluate the patients' consciousness, which may result in a designation as minimally conscious or vegetative.

BCI (Brain-Computer Interface) or BMI (Brain-Machine Interface): A device that directly records activity from the central nervous system and translates it into messages or commands.
FES (Functional Electrical Stimulation): A device that artificially stimulates muscles, such as muscles responsible for grasping or hand movements. FES systems controlled by BCIs can restore some movement to people with spinal cord injury or other conditions.

Invasive: A category of $\mathrm{BCI}$ in which electrodes are surgically implanted on or in the brain.

Neuroscience: The study of the human nervous system.

Non-invasive: A category of $\mathrm{BCI}$ that does not rely on invasive sensors. Most non-invasive BCIs use electrodes placed in an electrode cap, which is worn on the head.

Robotics: A category of engineering focused on developing, building, testing, and improving robots.

Single Switch BCI (ssBCI): A type of BCI that provides control equivalent to one single switch, such as an ON-OFF switch for a light.

Vegetative State (VS): A condition in which users are unable to produce goal-oriented responses to stimuli, among other deficits. 